\title{
MPACT Fast Neutron Multiplicity System Design Concepts
}

D. L. Chichester

S. A. Pozzi

J. L. Dolan

M. T. Kinlaw

A. C. Kaplan

M. Flaska

A. Enqvist

J. T. Johnson

S. M. Watson

October 2012

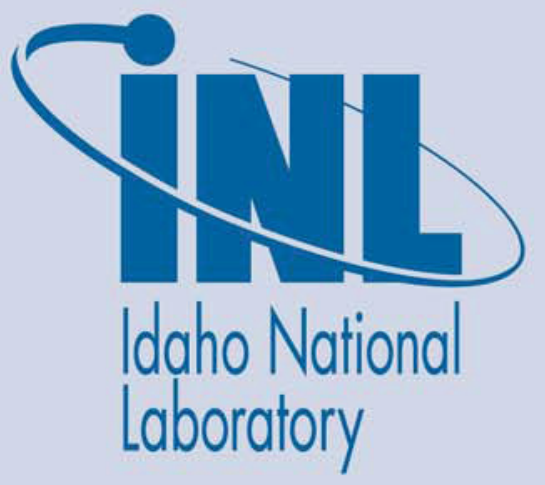

INL is a U.S. Department of Energy National Laboratory operated by Battelle Energy Alliance 
DISCLAIMER

This information was prepared as an account of work sponsored by an agency of the U.S. Government. Neither the U.S. Government nor any agency thereof, nor any of their employees, makes any warranty, expressed or implied, or assumes any legal liability or responsibility for the accuracy, completeness, or usefulness, of any information, apparatus, product, or process disclosed, or represents that its use would not infringe privately owned 


\section{MPACT Fast Neutron Multiplicity System Design Concepts}

D. L. Chichester ${ }^{1}$, S. A. Pozzi ${ }^{2}$, J. L. Dolan², M. T. Kinlaw ${ }^{1}$, A. C. Kaplan², M. Flaska ${ }^{2}$, A. Enqvist ${ }^{2}$, J. T. Johnson ${ }^{1}$, and S. M. Watson ${ }^{1}$

${ }^{1}$ Idaho National Laboratory

${ }^{2}$ Department of Nuclear Engineering \& Radiological Sciences, University of Michigan

October 2012

Idaho National Laboratory

Idaho Falls, Idaho 83415

http://www.inl.gov

Prepared for the

U.S. Department of Energy Office of Nuclear Energy Under DOE Idaho Operations Office

Contract DE-AC07-05ID14517 


\section{ACKNOWLEDGEMENTS}

The author's would like to acknowledge and thank Dr. Paul Hausladen and Dr. Jason Newby, both of Oak Ridge National Laboratory, for their assistance with the use of the Struck digitizer equipment, and for their thoughtful ideas and suggestions regarding this project.

The authors would like to kindly acknowledge the prior-year, in-kind support of Dr. Paolo Peerani, his research colleagues Dr. Alice Tomanin and Mr. Santino Frison, and other staff at the European Commission's PERLA Laboratory at the Joint Research Center in Ispra, Italy. Without his interest in our work, his desire to collaborate with our research team, and his support in hosting us at his laboratory, work that took place at JRC-Ispra in 2011 and 2012 would have been possible.

The authors would also like to acknowledge and thank the staff at INL's Materials and Fuels Complex and the ZPPR facility for their support and enthusiasm for the experiments described in this report. In particular, we would like to acknowledge the helpful assistance of shift supervisor Mr. Robert Neibert and his staff at ZPPR for their assistance in working to support experiments carried out at ZPPR.

The authors would also like to acknowledge and thank Dr. Mike Miller, technical director for the MPACT program, and Dr. Mark Mullen, former technical director for the MPACT program, for their support and enthusiasm of this research project.

The work in this report was sponsored by the U.S. Department of Energy's Fuel Cycle Research and Development program and its Materials Protection, Accounting, and Control Technologies (MPACT) program.

We would also like to acknowledge and thank the Nuclear Forensics Graduate Fellowship Program, which is sponsored by the U.S. Department of Homeland Security, Domestic Nuclear Detection Office and the U.S. Department of Defense, Defense Threat Reduction Agency, for supporting some of the activities of this project. 


\section{EXECUTIVE SUMMARY}

This report documents work performed by Idaho National Laboratory and the University of Michigan in fiscal year (FY) 2012 to examine design parameters related to the use of fast-neutron multiplicity counting for assaying plutonium for materials protection, accountancy, and control purposes. This project seeks to develop a new type of neutron-measurement-based plutonium assay instrument suited for assaying advanced fuel cycle materials. Some current-concept advanced fuels contain high concentrations of plutonium; some of these concept fuels also contain other fissionable actinides besides plutonium. Because of these attributes the neutron emission rates of these new fuels may be much higher, and more difficult to interpret, than measurements made of plutoniumonly materials. Fast neutron multiplicity analysis is one approach for assaying these advanced nuclear fuels.

Studies have been performed to assess the conceptual performance capabilities of a fast-neutron multiplicity counter for assaying plutonium. Comparisons have been made to evaluate the potential improvements and benefits of fast-neutron multiplicity analyses versus traditional thermal-neutron counting systems. Fast-neutron instrumentation, using for example an array of liquid scintillators such as EJ-309, have the potential to either a) significantly reduce assay measurement times versus traditional approaches, for comparable measurement precision values, b) significantly improve assay precision values, for measurement durations comparable to current-generation technology, or c) moderately improve both measurement precision and measurement durations versus current-generation technology. Using the MCNPX-PoliMi Monte Carlo simulation code, studies have been performed to assess the doubles-detection efficiency for a variety of counter layouts of cylindrical liquid scintillator detector cells over one, two, and three rows.

Ignoring other considerations, the best detector design is the one with the most detecting volume. However, operational limitations guide a) the maximum acceptable size of each detector cell (due to PSD performance and maximum-acceptable per-channel data throughput rates, limited by pulse pile-up and the processing rate of the electronics components of the system) and b) the affordability of a system due to the number of total channels of data to be collected and processed. As a first estimate, it appears that a system comprised of two rows of detectors $5 " \varnothing \times 3$ " would yield a working prototype system with excellent performance capabilities for assaying Pu-containing items and capable of handling high signal rates likely when measuring items with $\mathrm{Pu}$ and other actinides. However, it is still likely that gamma-ray shielding will be needed to reduce the total signal rate in the detectors. As a first step prior to working with these largersized detectors, it may be practical to perform scoping studies using small detectors, such as already-on-hand $3 " \varnothing \times 3 "$ detectors. 


\section{CONTENTS}

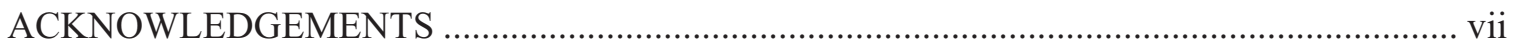

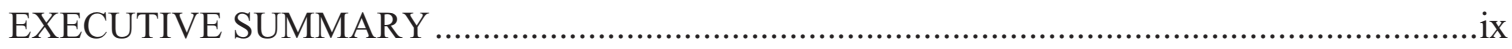

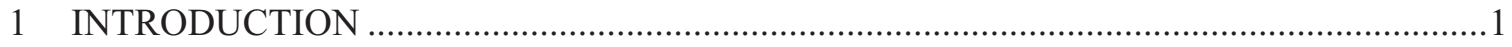

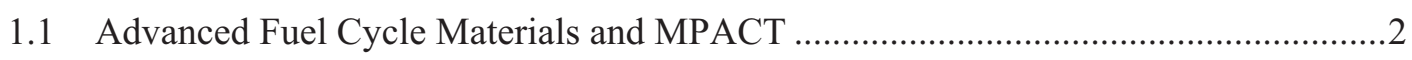

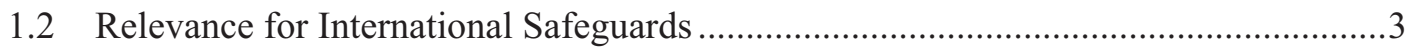

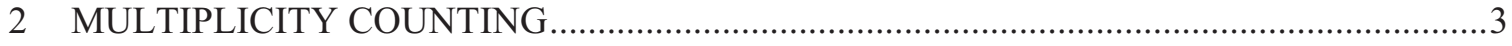

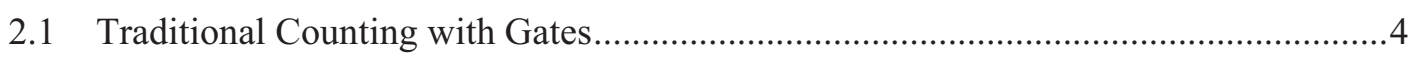

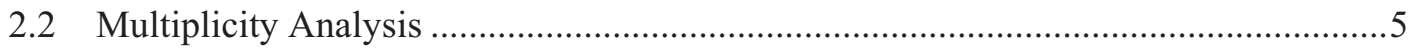

2.3 The Motivation for Fast-Neutron Counting .......................................................

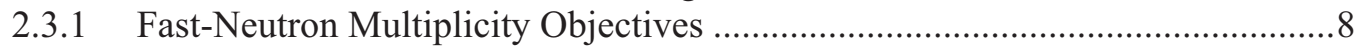

2.3.2 Motivation - Performance Improvement ............................................... 8

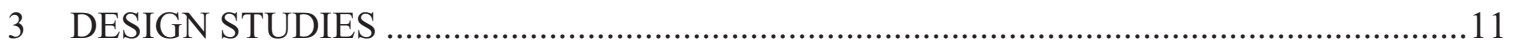

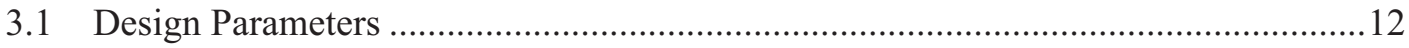

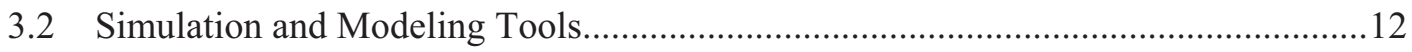

3.2.1 Simulating Multiplicity Systems with MCNPX-PoliMi/MPPost ......................13

3.2.2 Simulating Multiplicity and Using the PTRAC Output from MCNPX .............13

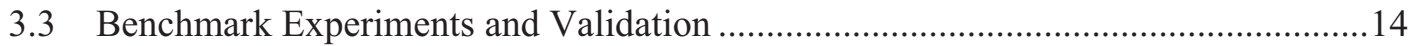

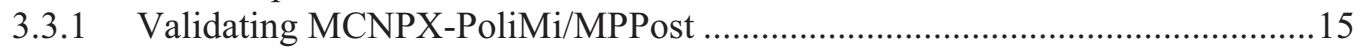

3.3.2 Sensitivity of a Fast-Neutron Multiplicity Counter............................................16

3.3.3 Comparing Direct MCNPX PTRAC Data with MCNPX-PoliMi ....................17

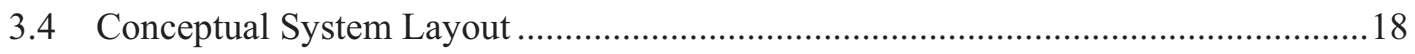

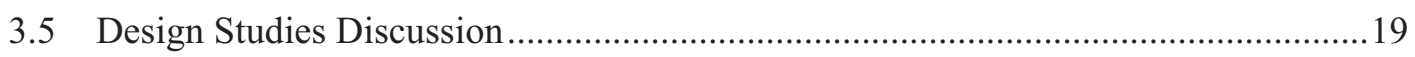

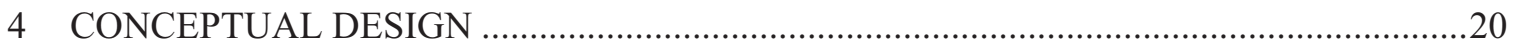

4.1 Expected Performance - 2 Rings of 5" $\varnothing \times 3$ " Detectors .............................................. 21

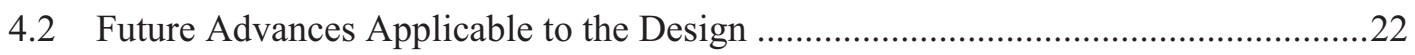

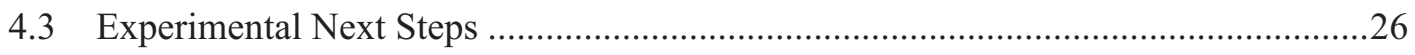

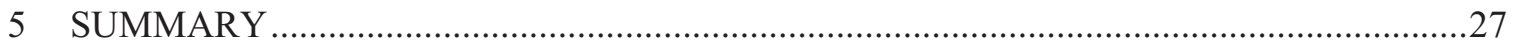

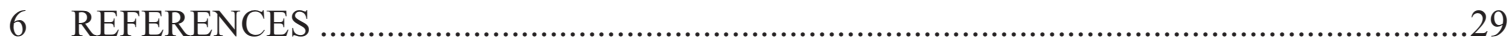

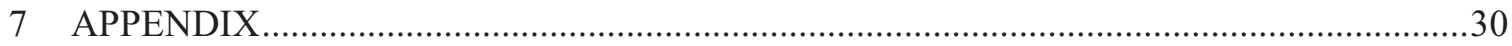




\section{FIGURES}

Figure 1 Calculated RSD (\%) versus sample mass for representative detection systems. Left: slow die-away system with $\tau=50 \mu \mathrm{s}, \mathrm{G}=1.257 \tau, \mathrm{P}_{\mathrm{d}}=1.5 \mu \mathrm{s}$, and $\varepsilon=$ 0.35 . Right: fast die-away system (solid lines) with $\tau=10 \mathrm{~ns}, \mathrm{G}=1.257 \tau, \mathrm{P}_{\mathrm{d}}=$ $1.5 \mathrm{~ns}$, and $\varepsilon=0.35$. Calculations for the longer die-away system (broken lines) are included for comparisons.

Figure 2 Calculated RSD (\%) versus detector die-away times for representative detection systems. Left: short predelay with $\mathrm{G}=1.257 \tau, \mathrm{P}_{\mathrm{d}}=1.5 \mathrm{~ns}$, and $\varepsilon=0.35$. Right: typical thermal neutron detection system predelay with $G=1.257 \tau, P_{d}=$ $1.5 \mu \mathrm{s}$, and $\varepsilon=0.35$.

Figure 3 Simulated neutron energy distributions (a) for neutron sources including plutonium metal, MOX, ${ }^{252} \mathrm{Cf}$, and AmBe. The simulated and measured energy dependent intrinsic neutron detection efficiencies (b) for 3"x3" and 5" 5 " liquid scintillation detectors.

Figure 4 Graphical interface for the PTRAC file reading program. The software produces both a user-friendly version of the original PTRAC file and a list-mode type data file for subsequent processing and/or data analysis.

Figure 5 A UM measurement system (a) with four $3 " \times 3$ " liquid scintillators, $0.1 \mathrm{~cm}$ lead shielding, and a CAEN DT5720 digitizer measuring approximately $60 \mathrm{~g}$ of plutonium. The neutron source contributions (b) for the $\mathrm{PuO}_{2}$ pellets measured at the JRC facility.

Figure 6 Simulated and measured ${ }^{255} \mathrm{Cf}$ pulse-height distributions (a) with an average point-by-point agreement error of $4.1 \%$. Photon discrimination (b) through PSD techniques at $70 \mathrm{keVee}$ light-output threshold (approximately $650 \mathrm{keV}$ neutron energy deposited).

Figure 7 Simulated and measured ${ }^{252} \mathrm{Cf}$ neutron multiplicity values (a) with agreement errors between 10 and 16\%. Simulated and measured neutron doubles (b) from three measurements of $\mathrm{PuO}_{2}$ pellets of increasing mass with statistical errors between 2 and $4 \%$.

Figure 8 The relationship between neutron singles and doubles with plutonium mass, measured with the partial UM measurement system.

Figure 9 Measured neutron pulse-height distributions (normalized to their integral) for

${ }^{252} \mathrm{Cf}$, mixed-oxide powder (MOX), and the $\mathrm{PuO}_{2}$ pellets.

Figure 10 Left: geometry setup for these simulations. The two cylinders are $\sim 93 \% \mathrm{HEU}$ castings, with a ${ }^{252} \mathrm{Cf}$ spontaneous fission source in the middle of the left casting. Cross correlations were simulated between detectors 1 and 3. Right: comparison of the output of a custom PTRAC reader and coincidence counting program with output from MCNPX-PoliMi.

Figure 11 Example of fast-neutron, multiplicity-counter models (a) JRC measurement system based on EJ-309 liquid scintillators, (b) a full ring (12 detectors) of 3" $\times$ 3" EJ-309 liquid scintillators, (c) two rings of 3" $\times 5$ " EJ-309 liquid scintillators, and (d) three rings of 5" $\times 2$ " EJ-309 liquid scintillators. The models include the active volume of the liquid scintillators, 0.1 " of lead shielding, and $\mathrm{PuO}_{2}$ pellets. 
Figure 12 The trend of simulated doubles rates with plutonium mass for 21 fast-neutron multiplicity counter designs. The number of detectors and the detector size were both varied. The figures compare the response according to the number of detector rings.

Figure 13 Plutonium mass statistical uncertainty for a 1-minute measurement with the two rings of $5 " \times 3$ " length design.

Figure 14 Plutonium mass uncertainty as a function of measurement time, for the three $\mathrm{PuO}_{2}$ pellet cases simulated with the two rings of 5 " $\times 3$ " length design.

Figure 15 Three, LAN controllable, 16-channel high-voltage boards contained in an MPod mini-crate for supplying a bias to a large quantity of detectors.

Figure 16 Three, time-synchronized CAEN V1720 digitizers (encompassing 24 detector channels) where information is passed via optical links.

Figure 17 A pulse-shape discrimination method that can be automated to perform more accurate photon and neutron discrimination across the entire range of pulse heights measured in the liquid scintillators.

Figure 18 An eight-channel Struck SIS3302 8-channel digitizer card under evaluation at INL.

Figure 19 Test activities at INL to evaluate the performance of a square-shaped liquidscintillator filled with EJ-309 material.

Figure 20 MOX fuel pins available for measurement and fast neutron multiplicity counter calibration at INL.

\section{TABLES}

Table 1 The doubles-rate sensitivity for the designs presented in Figure 12. 


\section{MPACT Fast Neutron Multiplicity System Design Concepts}

\section{INTRODUCTION}

Idaho National Laboratory (INL) has been working to explore new methods for analyzing nuclear materials using fast, time-correlated measurements for several years.[1,2] This work, supported by the U.S. Department of Energy's Fuel Cycle Research and Development program and its Materials Protection, Accounting, and Control Technologies (MPACT) program, has been a collaborative effort including staff at INL as well as staff and students in the Department of Nuclear Engineering \& Radiological Sciences at the University of Michigan (UM). These activities have included simulation and modeling using the MCNP-PoliMi Monte Carlo simulation tool and experiments to validate the simulations, development of hands-on experimental methods, and the discovery of pitfalls and challenges in performing these types of measurements that cannot be identified any other way. INL possess a strong background in theses areas, notably addressing nuclear security and safeguards challenges, heavily weighted towards real world experiments and system-level development and demonstration efforts, and the use of ENGs in active neutron interrogation. The University of Michigan team is a recognized world leader in the study and development of the MCNP-PoliMi computer code for modeling time-correlated measurements, as well as in the use of liquidscintillator-based detector systems for studying and characterizing special nuclear materials and their time-correlated signatures.

This report documents work performed by INL and UM in fiscal year (FY) 2012 to examine design parameters related to the use of fast-neutron multiplicity counting for assaying plutonium for materials protection, accountancy, and control purposes. This project seeks to develop a new type of neutron-measurement-based plutonium assay instrument suited for assaying advanced fuel cycle materials. Some current-concept advanced fuels contain high concentrations of plutonium; some of these concept fuels also contain other fissionable actinides besides plutonium. Because of these attributes the neutron emission rates of these new fuels may be much higher, and more difficult to interpret, than measurements made of plutonium-only materials. The most commonlyused approach for assaying plutonium is the use of thermal-neutron coincidence and multiplicity counters. However, these instruments can have difficulty when analyzing high-rate neutron sources. Also, there is a strong desire to develop new, alternative plutonium-assay systems that do not rely on the use of ${ }^{3} \mathrm{He}$ detectors (as are most-often used in thermal neutron systems) due to recent shortages of this material for safeguards applications.

A promising approach in this area is to perform measurements at much faster (nanosecond) time scales versus the longer (microsecond) time scales of thermal neutron systems. Fast-neutron measurements dramatically reduce the negative impacts of random coincidences in correlated-neutron assays that can occur with high count-rate samples. They also allow for in-depth analysis of multiplication phenomena in sample items in comparison with thermal analysis systems. Lastly, faster assay systems have the 
potential to reduce the burden on facility operators by reducing measurement times and improving the precision of assay measurements.

The long-term goals of this project are to design and build a fast neutron multiplicity analysis system for assaying advanced fuel cycle materials and then to test and evaluate this instrument using these materials. This project supports Objective 4 from the Nuclear Energy Research and Development Roadmap to "minimize the risks of nuclear proliferation and terrorism" and more specifically the Advanced Instrumentation sub-item in the Safeguards and Physical Security Technologies and Systems area "Development of advanced passive detectors such as neutron multiplicity counting."[3]

\subsection{Advanced Fuel Cycle Materials and MPACT}

As described in the FY2009 and FY2011 End-of-Year Reports, advanced nuclear fuels are currently under development within the Department of Energy's Fuel Cycle Research and Development program as part of a long-term research effort focused at understanding the behavior of mixed-oxide (MOX) fuels containing minor actinides and long-lived fission products.[1,2] The aim of this work is to understand how these materials impact the long-term performance of nuclear fuel in order to be able to design and manufacture advanced fuels for use in next-generation reactors. Reusing, or recycling, the higher actinides and long-lived fission products in advanced nuclear fuels ultimately leads to the transmutation of these materials into shorter-lived waste products which may be more easily and more safely disposed of. There are several potential benefits of reusing nuclear fuel including the reclamation of additional energy content from once-through used fuels, the reduction or removal of longer-lived waste products from spent fuel, and the lessening of the storage demands eventually placed on facilities for the long-term storage or disposal of spent fuels. In parallel with the fuel development projects research and development is also underway to develop advanced fuel reprocessing approaches to produce these fuels and to develop advanced reactors to use these fuels. However, in addition to these core engineering research and development projects the ultimate viability of these new technology developments will be critically linked to advances in nuclear safeguards and material protection, accounting, and control technologies (MPACT).

Traditional nuclear safeguard measurement techniques used to monitor uranium oxide fuels are not well-suited for analyzing advanced MOX fuels. Gross gamma-ray counting is complicated by the presence of the additional radioactive materials in the fuel while high-resolution gamma-ray spectroscopy can be difficult to perform due to the presence of multiple interferences associated with the presence of the minor actinides. Similarly, the powerful passive and active neutron-based nondestructive assay techniques used with current-generation fresh and irradiated commercial nuclear fuel are complicated by the presence of multiple higher actinides, some of which have spontaneous fission and induced fission signatures comparable to plutonium. From 2009 through 2012 it has been the goal of the INL-UM collaboration to explore techniques for fast-neutron and photon-correlation measurements, both passively and with active interrogation. The aim of these efforts has been to improve the fundamental understanding of nuclear materials and the physics of detection methods through coupled theory, simulation, and experiment, as necessary to develop next-generation materials management and MPACT technology. More broadly speaking, these efforts have been 
part of the larger MPACT research portfolio seeking to enhance overall nuclear fuel cycle proliferation resistance via improved technologies for used fuel management.

Important aspects of long-term, science-based, engineering-driven research and development (R\&D) include small-scale experiments, theory development, and advanced modeling and simulation with validation experiments. This project embraces this paradigm for the "science-based" R\&D approach for improving domestic MPACT approaches for security and safeguards.

\subsection{Relevance for International Safeguards}

Nuclear safeguards are defined as the effort to prevent diversion of fissile material. In 1970, the Treaty on the Nonproliferation of Nuclear Weapons (NPT) was entered into force with the objective to prevent the spread of nuclear weapons and their technology, while encouraging the peaceful use of nuclear technology.[4] Through the treaty a safeguards system was established. Nuclear safeguards endeavors are the responsibility of the International Atomic Energy Agency (IAEA) and are supported by diplomatic and economic means.

Given the increase in nuclear facilities across the world and innovation in the nuclear fuel cycle, new technology is needed to continue special nuclear material (SNM) accounting, control, and safeguards efforts. Specifically, the planned increase in fuel reprocessing warrants innovation in novel safeguards techniques to minimize the associated nonproliferation risks. The national energy policy has recommended research efforts in the development of reprocessing and fuel treatment technologies that are more proliferation-resistant. Additionally, safeguards designed directly into a new facility will be essential to international safeguards success.

\section{MULTIPLICITY COUNTING}

Nuclear safeguards rely on technology used during nuclear facility inspections to detect any diversion of fissile material. The most standard form of safeguards confirms the presence and type of materials from a facility's declarations. The technologies used to verify the material declarations include both destructive and nondestructive assay. Nondestructive assay is a preferred method of investigation and can include technologies based on neutron, photon, or calorimetric measurements. All of these concepts have their pros and cons, but neutron measurements remain to be a leading method. Neutrons are more penetrating than other forms of radiation and are they are less prevalent in radiation background and naturally occurring radioactive materials. An added benefit of neutron measurements exists due to the emission of multiple neutrons spontaneously from a single reaction, which is unique to fission. Therefore, neutron multiplicity measurements, where the neutron multiplicity distributions are measured, have continued to rise to the top for characterizing fissile materials in nuclear accountancy applications.

Early characterization systems measured only the neutron rate, which was applicable to only a few types of plutonium containing materials, considering there are other neutron emitting reactions present in many plutonium containing materials. Further developments extended systems into neutron coincidence counters, which provided a method to isolate only the measurement of neutrons from fission and has been extensively applied in safeguards. With the measurement of the neutron fission rate and knowledge of the neutron multiplicity distribution, the mass of certain plutonium isotopes 
can be identified. Neutron coincidence counting has not been as applicable to domestic accountability considering that only two parameters are measured (singles and doubles) and therefore the system's neutron detection efficiency must be known. For impure plutonium samples, the neutron detection efficiency of the system may change and become a variable due to the effects of neutron scattering and/or moderation within the sample. To solve this problem, assumptions must be made regarding the amount of $(\alpha, n)$ neutrons or the sample multiplicity. For greater accuracy and the minimization of assumptions, neutron multiplicity systems were developed that provide three measured parameters: singles, doubles, and triples. With a neutron multiplicity system, the goal is to be able to correctly characterize any nuclear fuel cycle material without any knowledge of the material's matrix.[5]

Currently available multiplicity systems are categorized based on the range of plutonium mass they are designed to quantify. For lower masses of plutonium (0.1 to $500 \mathrm{~g}$ of plutonium) low-level inventory sample coincidence counters are available from companies like Canberra.[6] High-level systems measure up to several kilograms of plutonium. Both low and high level systems contain just fewer than twenty ${ }^{3} \mathrm{He}$ tubes. These systems rely on spontaneous fission from the even numbered isotopes of plutonium. Similar systems, such as active-well coincidence counters, can quantify uranium as well but require a neutron active-interrogation source and more than twice as many ${ }^{3} \mathrm{He}$ tubes. Other systems are designed to measure specific nuclear fuels such as neutron coincidence collars (PWR, BWR, CANDU assemblies), fast-breader reactor subassembly counters (single or groups of fast-breader fuel pins), and plutonium scrap counters (impure plutonium samples or MOX). A fast neutron multiplicity counter described and discussed in this report is applicable for all of these measurement scenarios.[7]

\subsection{Traditional Counting with Gates}

In traditional ${ }^{3} \mathrm{He}$ systems, when fission occurs in the measured sample and neutrons are emitted, they are moderated in an optimized polyethylene medium and the neutron population exponentially dies away. Neutrons are removed from the detector system by escaping the system, neutron absorption in nearby neutron absorbing materials (such as hydrogen or plutonium), or ideally neutron capture by ${ }^{3} \mathrm{He}$. Typical die-away times for ${ }^{3} \mathrm{He}$ systems are on the order of tens of micro-seconds but are practically nonexistent for fast neutron counters. For each neutron detection, a ${ }^{3} \mathrm{He}$ detector provides a single pulse that is fed through a system of electronics resulting in a single stream of pulses for all of the detectors present in the system. It is then necessary to separate the correlated neutron events (fission events providing the plutonium signature) from the uncorrelated neutron events (events from other neutron emitting reactions and background events). This is done through optimized time-gating of the time-dependent pulse stream.[5]

A common method for identifying correlated events is through shift-register circuits based on the concept of a Rossi-alpha distribution.[5] The Rossi-alpha distribution is the distribution in time of events that occur after a randomly chosen start event. This distribution will be constant with time if only uncorrelated events are detected and therefore will have features when correlated events are present. The distribution is defined by the constant uncorrelated events plus the exponentially 
decaying "Real" or correlated events. Time gates are then defined to isolate the "Reals + Accidentals" portion and the "Accidentals" portion of the distribution. The "Reals + Accidentals" gate will be on the order of tens of micro-seconds, and then there will be a long delay (on the order of thousands of micro-seconds) before the "Accidentals" gate is opened for a time more similar to the initial gate. An actual measured distribution will not increase exponentially as you take the limit to zero, due to pulse pile-up and electronic dead-time effects. Therefore, a "pre-delay" time gate is also specified to correct for these limiting effects.

Identification of only the "Reals" leads to the indication of the multiplicity distribution and furthermore the fission rate, which is necessary to determine the plutonium mass.[5] Specialized electronics exist to take the stream of pulses and isolate the time-gates to identify the neutron multiplicity distributions for both the "Reals + Accidentals" and "Accidentals" gates. The result of analyzing and unfolding both sets of data is the singles, doubles, and triples values needed for eventual mass quantification.[5] A fast neutron multiplicity counter can directly provide these three parameters without the circuitry and unfolding.

\subsection{Multiplicity Analysis}

One of the primary purposes of utilizing neutron multiplicity counting over traditional neutron coincidence counting lies in the extension of possible parameters that can be determined. With coincidence counting, $\mathrm{m}_{240 \mathrm{eff}}$, the ${ }^{240} \mathrm{Pu}$ effective mass (i.e., the mass of ${ }^{240} \mathrm{Pu}$ that will produce a coincidence rate equivalent to the sum of all even isotopes in the sample) is determined according to the following equation:

$$
\mathrm{m}_{240 \mathrm{eff}}=2.52 \mathrm{~m}_{238}+\mathrm{m}_{240}+1.68 \mathrm{~m}_{242}
$$

Eq. 1

where $\mathrm{m}_{238}$ is the mass of ${ }^{238} \mathrm{Pu}$ in the sample, $\mathrm{m}_{240}$ is the mass of ${ }^{240} \mathrm{Pu}$ in the sample, and $\mathrm{m}_{242}$ is the mass of ${ }^{242} \mathrm{Pu}$ in the sample.[5] However, in cases where additional parameters are sought, such as the $(\alpha, n)$ reaction rate, additional information is also required. For multiplicity counting, this additional information is gleaned by measuring, in addition to the first and second moments, the third moment of the detected neutron distribution. With these three moments, the sample multiplication, fission rate, and $(\alpha, n)$ reaction rate can each be calculated.

The detected neutron singles rate, $\mathrm{S}$, represents all neutrons detected, regardless of their reaction of origin, including those emitted via spontaneous fission, induced fission, and $(\alpha, n)$ reactions. Empirically, this rate can be calculated as: 


$$
\mathrm{S}=\mathrm{F} \varepsilon \mathrm{M} v_{\mathrm{s}, 1}(1+\alpha)
$$

Eq. 2

where $\mathrm{F}=$ fission rate $\left(\sim \mathrm{m}_{240} \cdot 10^{3} / \mathrm{v}_{\mathrm{s}, 1}\left[\mathrm{~s}^{-1} \mathrm{~g}^{-1}\right]\right), \varepsilon=$ detection efficiency, $\mathrm{M}=$ sample multiplication (leakage), and $\alpha=$ the ratio of $(\alpha, n)$ neutrons to spontaneous fission neutrons.[5,8,9]" For this report, factorial moments with subscript "s" denote spontaneous fission, while those without this marking correspond to induced fission. The detected neutron doubles rate, $\mathrm{D}$, is dependent on spontaneous fission, induced fission, and $(\alpha, n)$ reactions. However, the spontaneous fission and $(\alpha, n)$ terms depend on the second moments, and the induced fission term relates to induced fissions resulting from the multiplication of spontaneous fission neutrons.

$$
\mathrm{D}=\frac{\mathrm{F} \varepsilon^{2} \mathrm{f} \mathrm{M}^{2}}{2}\left[v_{\mathrm{s}, 2}+\left(\frac{\mathrm{M}-1}{v_{1}-1}\right) v_{\mathrm{s}, 1}(1+\alpha) v_{2}\right]
$$

The expression for the doubles rate is given in Eq. 3, where $\mathrm{f}$ is the gate fraction, as shown in Eq. 4, where $P_{d}$ is the counting gate pre-delay, $\tau$ is the detector die-away period (1/e decay period), and $\mathrm{G}$ is the counting gate width.

$$
\mathrm{f}=\mathrm{e}^{-\mathrm{P}_{\mathrm{d}} / \tau}\left(1-\mathrm{e}^{-\mathrm{G} / \tau}\right)
$$

Regarding the triples rate, $\mathrm{T}$, many different processes can contribute to the detection of three neutrons within a counting interval. The empirical representation of this rate is given by:

$$
\mathrm{T}=\frac{\mathrm{F} \varepsilon^{3} \mathrm{f}^{2} \mathrm{M}^{3}}{6}\left[v_{\mathrm{s}, 3}+\left(\frac{\mathrm{M}-1}{v_{1}-1}\right)\left[3 v_{\mathrm{s}, 2} v_{2}+(1+\alpha) v_{\mathrm{s}, 1} v_{3}\right]+3\left(\frac{\mathrm{M}-1}{v_{1}-1}\right)^{2} v_{\mathrm{s}, 1}(1+\alpha) v_{2}^{2}\right]
$$

Eq. 5

In this form, an assumption is made where the detector die-away may be approximated, at a minimum, as a single exponential die-away. Hence, the triples gate fraction $f_{t}$ is simply the square of the doubles gate fraction. If this approximation does not suffice, a more appropriate expression for the triples gate fraction is provided by Eq. 6-6 in reference 5.

As stated above, the primary advantage of including the third moment of the detected neutron distribution is an ability to determine from the three measured moments

\footnotetext{
${ }^{*}$ Note: References 5,8, and 9 are applicable for all of the equations cited in this report.
} 
the sample multiplication, fission rate (thus the ${ }^{240} \mathrm{Pu}$ effective mass), and $(\alpha, n)$ reaction rate. The sample multiplication can be expressed as a cubic equation as:

$$
\mathrm{a}_{0}+\mathrm{a}_{1} \mathrm{M}+\mathrm{a}_{2} \mathrm{M}^{2}+\mathrm{a}_{3} \mathrm{M}^{3}=0, \quad \text { Eq. } 6
$$

where

$$
\begin{aligned}
& \mathrm{a}_{0}=-\mathrm{T}, \\
& \mathrm{a}_{1}=\mathrm{D}\left[\frac{v_{\mathrm{s}, 3}}{v_{\mathrm{s}, 2}}-\frac{3 v_{2}}{\left(v_{1}-1\right)}\right], \\
& \mathrm{a}_{2}=\mathrm{S}\left[\frac{v_{2} v_{\mathrm{s}, 3}}{\left(v_{1}-1\right) v_{\mathrm{s}, 2}}-\frac{v_{3}}{\left(v_{1}-1\right)}\right]+\frac{3 \mathrm{D} v_{2}}{\left(v_{1}-1\right)}, \text { and } \\
& \mathrm{a}_{3}=\mathrm{S}\left[\frac{v_{3}}{\left(v_{1}-1\right)}-\frac{v_{2} v_{\mathrm{s}, 2}}{\left(v_{1}-1\right) v_{\mathrm{s}, 2}}\right] .
\end{aligned}
$$

With the calculated $\mathrm{M}$, the fission rate is given according to Eq. 11.

$$
F=\frac{D-\frac{M(M-1) v_{2} S}{v_{1}-1}}{\varepsilon M^{2} v_{s, 2}}
$$

Similarly, the $(\alpha, n)$ reaction rate, $\alpha$, is given according to Eq. 12 .

$$
\alpha=\frac{\mathrm{S}}{\mathrm{MF} v_{\mathrm{s}, 1}}-1
$$

Here, the assumption is made that the detector efficiency can be measured and/or calculated based on calibrations with known fission sources (i.e., ${ }^{252} \mathrm{Cf}$ ). If this is not true, as may be the case for substantially-altered neutron spectra emitted from large matrices, then if the multiplication can be held at 1, $\alpha$ can be determined, followed by the fission rate, $\mathrm{F}$, and the neutron detection efficiency.[5] Also, multiplication bias correction and deadtime corrections are being neglected here.[5,10,11] 


\subsection{The Motivation for Fast-Neutron Counting}

At nuclear facilities, domestically and internationally, most measurement systems used for nuclear materials' control and accountability rely on ${ }^{3} \mathrm{He}$ detectors. These systems depend on well-established relationships to interpret multiplicity-type measurements for verifying quantities of SNM. Due to resource shortages, alternatives to ${ }^{3} \mathrm{He}$ systems are urgently needed. Additionally, in the near term, the cost of current ${ }^{3} \mathrm{He}$ based systems continues to increase as the supply cannot meet the demand. This mission also presents the opportunity to broaden the capabilities of these types of measurement systems to improve current multiplicity techniques and expand the scope to encompass advanced nuclear fuels.

\subsubsection{Fast-Neutron Multiplicity Objectives}

Within this material protection, accounting, and control technology project, INL and UM are working together to design a fast-neutron multiplicity counter with organicliquid scintillators to quantify fissile material mass. With excellent timing properties of liquid scintillators in conjunction with excellent neutron/photon pulse-shape discrimination (PSD), we are designing a multiplicity system that is less prone to detection/characterization errors for high-activity nuclear materials. Due to the direct measurement of fast neutrons from fission, supplementary quantities related to the fission neutron's energy can be also utilized. Also, an organic-liquid scintillation multiplicity system can make use of photon and joint neutron and photon multiplicities to solve for additional unknowns.

The INL and UM contributors have many years of experience with liquid scintillators to measure SNM. The multi-disciplinary design efforts include: state-of-theart neutron/photon PSD techniques, advances in digital data-acquisition and fieldprogrammable-gate-array systems (on-the-fly data processing), automated detector gain matching techniques, and novel data-processing techniques.

\subsubsection{Motivation - Performance Improvement}

Fast-neutron counting may have several advantages over the thermal and epithermal neutron counters currently used for nondestructive assay of plutonium-bearing packages. Short die-away times $(\sim 10 \mathrm{~ns})$ allow assay of higher-order multiplicity with fewer random events, assays of samples with high $(\alpha, n)$ source terms, and assays using active interrogation sources. Inspection times required may be significantly reduced while maintaining acceptable measurement precision, higher-throughput operations may be supported, and the faster detector response times may allow for analysis of materials with substantially-higher emission/count rates.

Employing thermal and/or epithermal neutron detectors for coincidence or multiplicity counting typically requires that the emitted neutrons be moderated prior to reaching a detector's active region. Reducing the average fission neutron energy $(\sim 1-2$ $\mathrm{MeV}$ ) to a level at which the necessary capture reaction has a greater probability of occurrence consequently removes much, if not all, of the emission timing information from consideration. Assuming an "optimal" counting gate width that is on scale with detector die-away $(\mathrm{G}=\sim 1.26 \tau)$ is utilized, a detection system with $\tau=50 \mu$ s would have a gate width of $\sim 63 \mu \mathrm{s}$. [5] Comparing this setting with a theoretical fast neutron-based system $(\tau=50 \mathrm{~ns}, \mathrm{G}=63 \mathrm{~ns})$, the system with a long die-away would be subject to as 
many as three orders of magnitude more accidentals than would the fast die-away system. Further, a system that operates on timescales comparable with the timescale of fission chain production also allows for the resolution of uncertainties in multiplication and detection efficiency.[12] An additional disadvantage of moderating neutrons prior to their detection is the initial energy information of the detected neutron is lost. With a scintillator-based system, or similar fast neutron-based system, at least some portion of the neutron's energy information is retained. By combining an energy discrimination capability with the aforementioned short counting gate width, items with elevated $(\alpha, n)$ to-spontaneous fission ratios can be assayed in reasonable time periods; the potential also exists for improved signal-to-background ratios for active interrogation.[13]

To illustrate the comparison between fast neutron and thermal neutron-based systems a computer code based on the well-known "Ensslin Figure-of-Merit" algorithms was developed to calculate assay uncertainties for various system parameters.[14] Some example results from this code are illustrated in Figure 1, showing (left) the calculated relative standard deviation (RSD) as a function of sample mass for a representative detection system.
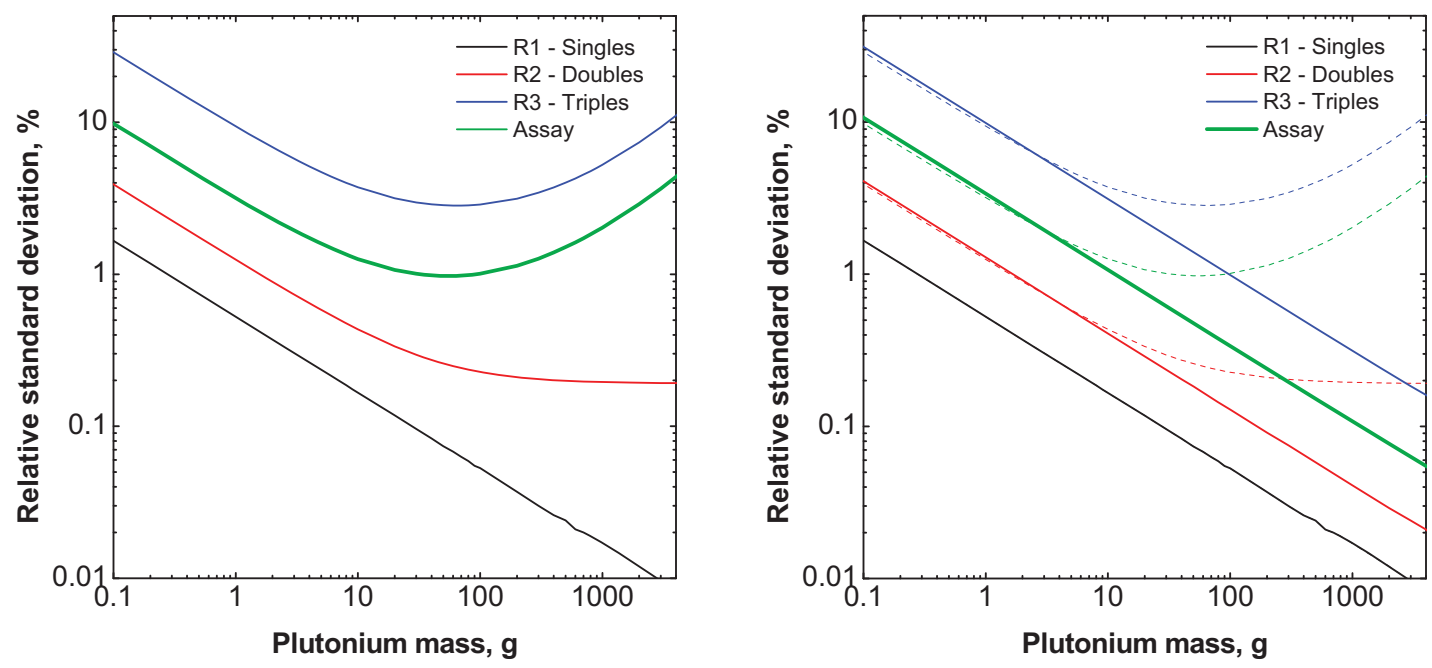

Figure 1 Calculated RSD (\%) versus sample mass for representative detection systems. Left: slow die-away system with $\tau=50 \mu \mathrm{s}, \mathrm{G}=1.257 \tau, P_{\mathrm{d}}=1.5 \mu \mathrm{s}$, and $\varepsilon=$ 0.35. Right: fast die-away system (solid lines) with $\tau=10 \mathrm{~ns}, G=1.257 \tau, P_{d}=1.5$ ns, and $\varepsilon=0.35$. Calculations for the longer die-away system (broken lines) are included for comparisons.

Calculation of measurement precision for the individual singles, doubles, and triples rates, as well as the total assay precision, were completed for a range of sample masses. The count time was set at $1000 \mathrm{~s}, \tau=50 \mu \mathrm{s}, \mathrm{G}=1.257 \tau, \mathrm{P}_{\mathrm{d}}=1.5 \mu \mathrm{s}$, and $\varepsilon=$ 0.35. At lower masses the spontaneous emission rate is relatively low; hence, the detection/count rate is also low. As a result, accidental coincidences are minimal and the $\mathrm{RSD}$ is dominated by detector efficiency. At higher masses the count rate correspondingly increases, leading to a substantial increase in the number of accidental coincidences. In this case, the RSD is heavily influenced by the detector die-away. In 
contrast, Figure 1 (right) presents equivalent RSD calculations for a faster die-away system (solid lines) with $\tau=10 \mathrm{~ns}, \mathrm{G}=1.257 \tau$, and $\mathrm{P}_{\mathrm{d}}=1.5 \mathrm{~ns}$. This plot includes the longer die-away RSD results (broken lines) for comparison. Higher-throughput is much less of an issue due to a significantly reduced number of accidental coincidences over the same measurement time. Quantitatively, for a $20 \mathrm{~g}{ }^{240} \mathrm{Pu}$ sample mass, the RSD for the $50 \mu$ s die-away time is $2.9 \%$, while the RSD for $\tau=10 \mathrm{~ns}$ is only $0.3 \%$. For equivalent count times the faster die-away system's RSD is an order of magnitude less than the slower system. Below $20 \mathrm{~g}$, for these particular sets of detector parameters, the separations between the RSD's become smaller until they are essentially equal (below $\sim 1$ g). In contrast, for masses above $20 \mathrm{~g}$, the separation continues to increase.

Aside from neutron detection efficiency, the die-away time of the detector is perhaps the most critical component of the multiplicity counter.[14] Simply stated, a detection system with a minimal die-away time allows for a correspondingly short counting gate width, and, thus, fewer accidental coincidences. RSD's for several sample cases as a function of detector die-away time are shown in Figure 2. For $\tau=70 \mu \mathrm{s}$, increasing the sample mass from $20 \mathrm{~g}(\mathrm{M}=1)$ to $200 \mathrm{~g}(\mathrm{M}=1.2)$, while maintaining $\alpha=$ 1 , only degrades the assay precision by a factor of $\sim 2$. However, with the same sample mass of $20 \mathrm{~g}$, increasing $\alpha$ to 10 results in an RSD $\sim 23$ times larger than the $\alpha=1$ case. Similarly, the RSD for the $200 \mathrm{~g}, \alpha=1$ case is $\sim 23$ times smaller than the $200 \mathrm{~g}, \alpha=10$ case. Clearly, increasing the $(\alpha, n)$ rate significantly degrades the assay RSD.

Despite the strong decrease in assay RSD with decreasing detector die-away, a sharp increase in the RSD for $\tau$ values below a few $\mu$ s is seen in the right panel of Figure 2. This trend is an artifact of maintaining a constant predelay, $\mathrm{P}_{\mathrm{d}}$, with varying detector die-away. With a typical shift register circuit, a predelay is employed to minimize artificial counting due to noise and pileup in the detector electronics. Mathematically, $\mathrm{P}_{\mathrm{d}}$ influences the assay RSD within the fraction of signal-triggered events, $\mathrm{E}_{\mathrm{k}}$, detected during the counting gate width $\left[\mathrm{E}_{\mathrm{k}} \propto\left(\mathrm{e}^{-\mathrm{Pd} / \tau}\right)^{\mathrm{k}}\right]$.[5] Hence, if $\mathrm{P}_{\mathrm{d}}$ is small relative to $\tau$, the sharp rise seen in the right panel of Figure 2 is not nearly as significant. For the data shown in the plot to the right, $\mathrm{P}_{\mathrm{d}}=1.5 \mu \mathrm{s}$. In contrast, the left panel of Figure 2 shows the corresponding assay RSD for a fast neutron detector as a function of die-away time (0 to $100 \mathrm{~ns}$ ), but with the predelay set to $1.5 \mathrm{~ns}$. Finally, comparing the data from both plots in Figure 2, for a $20 \mathrm{~g}$ sample $(\mathrm{M}=1, \alpha=1)$, using the fast neutron counter parameters improves the RSD by a factor of $\sim 10$ for the same counting period (1000 s). Or, if the same RSD is maintained, the required count time for the fast neutron system would decrease by a factor of $\sim 10$. 

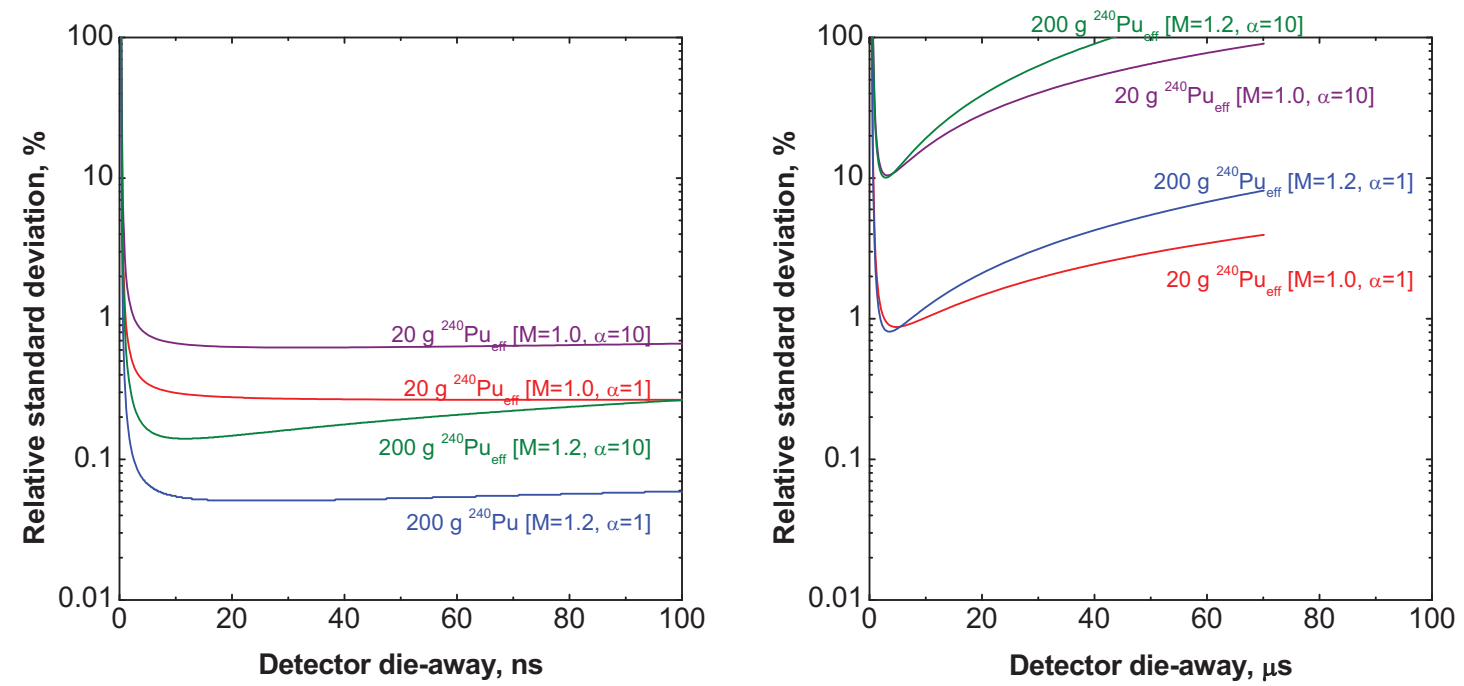

Figure 2 Calculated RSD (\%) versus detector die-away times for representative detection systems. Left: short predelay with $G=1.257 \tau, P_{d}=1.5 \mathrm{~ns}$, and $\varepsilon=0.35$. Right: typical thermal neutron detection system predelay with $G=1.257 \tau, P_{d}=1.5$ $\mu \mathrm{s}$, and $\varepsilon=0.35$.

\section{DESIGN STUDIES}

We aspire to design a fast neutron multiplicity counter to replace and build upon current counter technologies. We will focus this effort first on passive low-level systems and will build towards higher-level systems and application specific systems from this design. When designing a neutron multiplicity counter there are a number of standard goals that need to be kept in mind. First of all, the system efficiency must be sufficient enough to measure not only neutron singles and doubles but also triples. For traditional ${ }^{3} \mathrm{He}$ systems, $40-60 \%$ is a common range to aim for, while many systems have efficiencies on the order of $10-30 \% .[5,6]$ Similarly, the next primary goal is minimizing electronic dead-time losses and detector die-away times in order to maintain an efficient system.[5] A fast neutron multiplicity system with fast liquid scintillation detectors is inherently advantageous regarding these two issues.

Next, to develop a system that can quantify plutonium mass in a wide array of material types, it is important that the efficiency is consistent across a reasonable range of neutron energies.[5] Detected neutrons will be from spontaneous and induced fission events, which have quite similar neutron energy distributions, but will also be from $(\alpha, n)$ reactions which can vary significantly in neutron energy distributions. Examples of simulated neutron energy distributions are shown in Figure 3a for a variety of neutron sources including SNM (mixed-oxide fuel (MOX) and small plutonium metal samples) and isotopic neutron sources $\left({ }^{252} \mathrm{Cf}\right.$ and $\left.\mathrm{AmBe}\right)$. A fast neutron system does not require optimized moderation for neutrons across a wide range of energies, which is an additional benefit. Liquid scintillation detectors are conveniently consistent over the range of most neutron energies from fission and $(\alpha, n)$ neutrons. Both simulated and measured intrinsic neutron efficiency curves are shown in Figure $3 b$ for three by three inch and five inch by 
five inch liquid scintillators. The larger volume of the $5^{\prime \prime} \times 5^{\prime \prime}$ detector lends to increased intrinsic efficiency while the shape of the efficiency curve is consistent between the two detector sizes. The simulations are more accurate for the $3 " \times 3$ " inch detector, likely due to more complex light attenuation and collection effects that are present with greater detector volumes. Current efforts are in place to model these effects.

a)

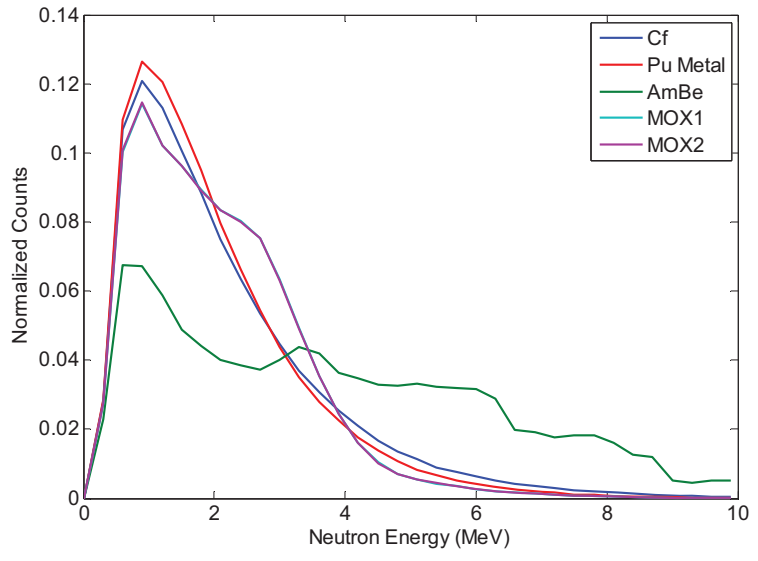

b)

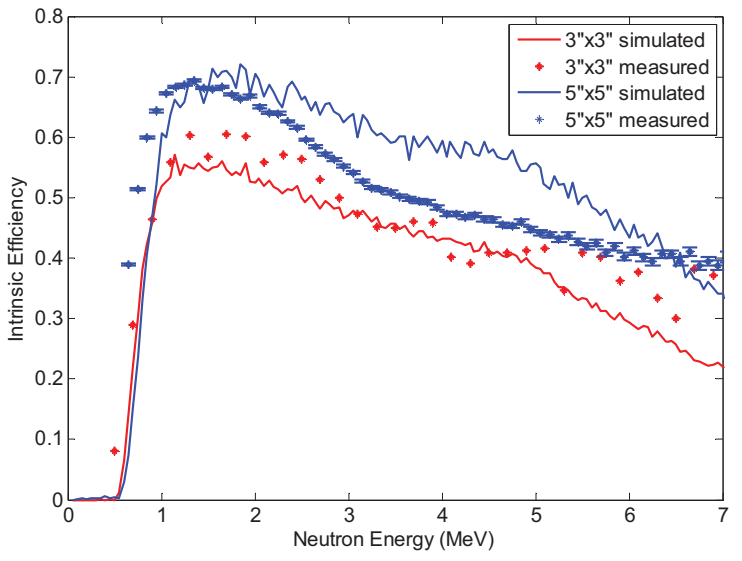

Figure 3 Simulated neutron energy distributions (a) for neutron sources including plutonium metal, MOX, ${ }^{252} \mathrm{Cf}$, and AmBe. The simulated and measured energy dependent intrinsic neutron detection efficiencies (b) for 3 "x3" and 5"x5" liquid scintillation detectors.

Lastly, when considering the design of the detector configuration, it is important to minimize the dependence of the system response to placement of the radioactive sample within the system.[5] In the field, it is not guaranteed that the distribution of the nuclear materials is known, and therefore the system must be relatively insensitive to reasonable placement errors. The dependence of the system to variation in sample placement can be investigated through simulation efforts.

\subsection{Design Parameters}

Considering the described design goals, there are a few parameters that are important to optimize in a fast neutron multiplicity system. These parameters include: detector diameter, detector depth, number of total detectors, number of detector rings, and detector placement. Detector gain and threshold are additional parameters that can be varied to impact the data analysis. During this optimization, the above goals are kept in mind while also considering effects like detector cross-talk and data acquisition limits. Through simulation of the total system response, all of these concepts were thoroughly investigated. Additionally, by meeting all of these design goals, traditional multiplicity mathematics is applicable to the fast neutron multiplicity design.

\subsection{Simulation and Modeling Tools}

Prior to experimental demonstration in the laboratory it is now standard practice to use computer modeling to predict the performance of material accountancy and 
safeguards instrumentation. Monte Carlo simulation tools are also the standard resource used for designing and optimizing these instruements. For this project the majority of research this has centered on the use of the UM MCNPX-PoliMi code for these activities. MCNPX-PoliMi is ideally suited for the design and optimization of neutron multiplicity instruments because of its inherent ability to support the analysis of multi-particle, timecorrelated events. Work at INL this year has also progressed towards developing the capability for extracting multi-particle, time-correlated data from MCNPX directly, using the PTRAC output.

\subsubsection{Simulating Multiplicity Systems with MCNPX-PoliMi/MPPost}

Several designs are being investigated using the MCNPX-PoliMi transport code and the MPPost post-processing code.[15,16] MCNPX-PoliMi is ideal for designing a fast-neutron multiplicity counter due to its: capability of realistically simulating correlated source events, detailed particle interaction output, and incorporation of SNM sources with accurately sampled energy, number of particles emitted, and their angle distributions. It is important that the physics of particle emission (specifically fission) is as accurate as possible when modeling correlated/multiplicity measurements.[17] MCNPX-PoliMi incorporates neutron and photon multiplicity distributions with correlated neutron and photon production.[15] After the production of all source particles, detailed interaction information is recorded within all volumes of interest. This detailed information can be processed to develop detector and measurement system response.

MPPost (a MCNPX-PoliMi post-processing code) processes the MCNPX-PoliMi data file into both individual detector and total system design responses. MPPost requires the output from MCNPX-PoliMi and the definition of various detector and measurement system parameters.[16] For a liquid scintillation detector system, some of these parameters include: particle energy to light-output conversion functions, detector pulse generation time, detection thresholds, dead-times, and particle correlation time windows. Outputs from MPPost include pulse-height distributions, correlated particle analysis such as time-of-flight and cross-correlation functions, and neutron and photon multiplicity.[16]

Both MCNPX-PoliMi and MPPost are available through the Radiation Safety Information Computation Center.

\subsubsection{Simulating Multiplicity and Using the PTRAC Output from MCNPX}

Custom software was written with the primary purpose of extracting user-selected information from the PTRAC file generated by MCNPX; a screen shot of an interface dialogue box is shown in Figure 4. In its current form, the program takes as input the PTRAC file and produces either a more readable text version of the original PTRAC data or a list-mode type output file. With the current version of MCNPX (2.7) there are as many as four different formats for the PTRAC file. As such, the program requires selection of which particular format type is to be read, including the number of header lines in the file. There are also options for selecting only specific interaction types (i.e., collisions/scattering, termination/capture, etc.); hence, by selecting in which cells the selected event types are to be tracked, a filtered list-mode data file is produced that is available for post-processing and data analysis. The rationale for this format was to 
enable the modeled data to be generated as close to the experimental data as possible for realistic comparisons.

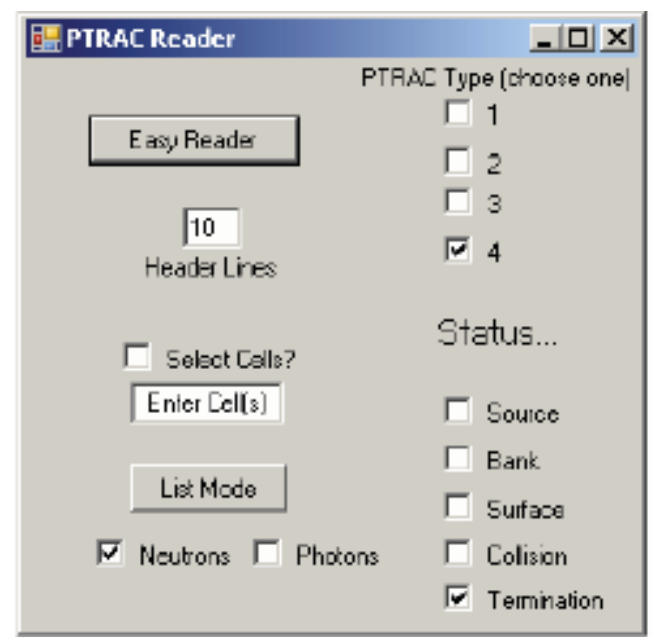

Figure 4 Graphical interface for the PTRAC file reading program. The software produces both a user-friendly version of the original PTRAC file and a list-mode type data file for subsequent processing and/or data analysis.

\subsection{Benchmark Experiments and Validation}

Measurements were performed on fissile materials at the Joint Research Centre (JRC) in Italy in April of 2012. A UM measurement system (Figure 5a) was utilized and the results were then replicated with MCNPX-PoliMi. In addition, data-analysis algorithms were tested for potential and limitations. Comparing the measured and simulated results built confidence in the use of MCNPX-PoliMi/MPPost for developing the proposed system design. Combinations of $\mathrm{PuO}_{2}$ pellets (also in Figure 5a) were measured to asses a plutonium mass sensitivity metric. The measured plutonium masses ranged from 20 to $60 \mathrm{~g}$ and the neutron emission contributions are as shown in Figure 5b. The materials measured are similar to those measured in low-level plutonium sample inventory counters and helped provide insight into how the detectors would respond to such materials. 

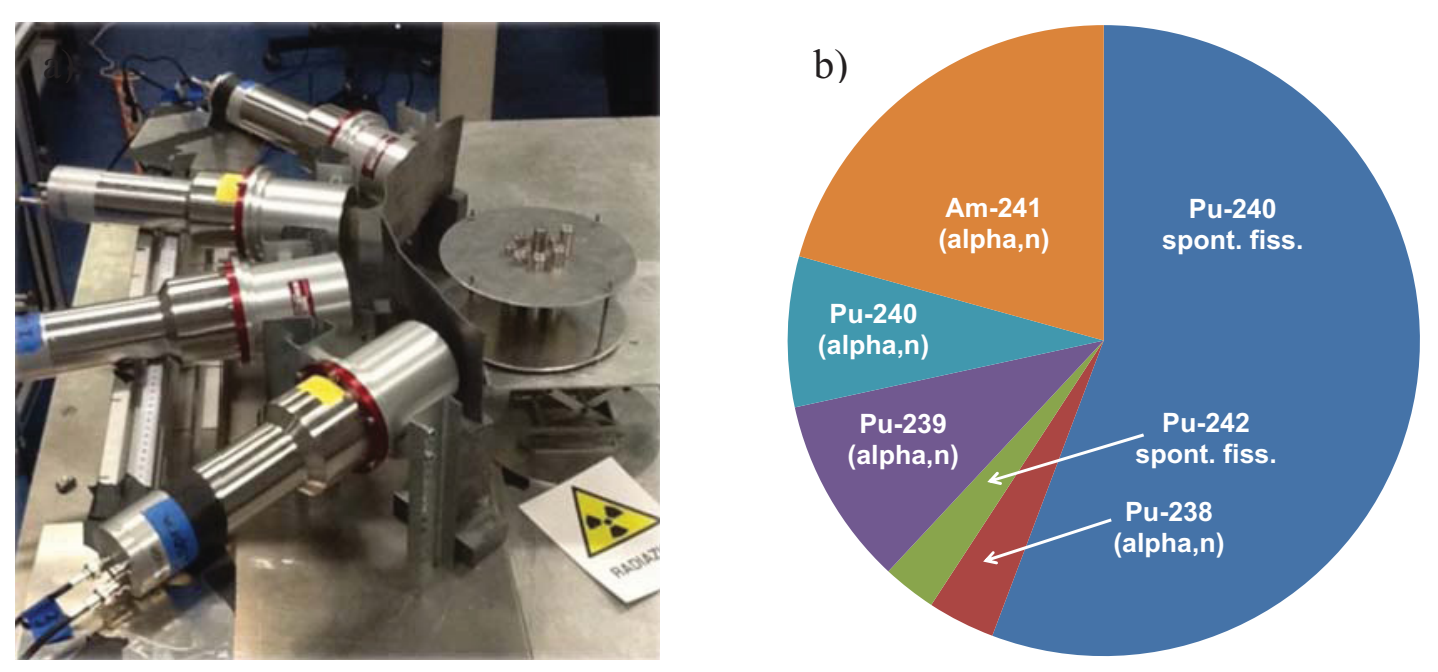

Figure 5 A UM measurement system (a) with four 3" $\times 3$ " liquid scintillators, 0.1 cm lead shielding, and a CAEN DT5720 digitizer measuring approximately $60 \mathrm{~g}$ of plutonium. The neutron source contributions (b) for the $\mathrm{PuO}_{2}$ pellets measured at the JRC facility.

\subsubsection{Validating MCNPX-PoliMi/MPPost}

Good agreements between the simulated and measured neutron pulse-height distributions and neutron multiplicity values measured from a ${ }^{252} \mathrm{Cf}$ source are shown in Figure $6 \mathrm{a}$ and Figure 7a. The measured results have undergone PSD (Figure 6b) to isolate the neutron pulses. With a more complex neutron source $\left(\mathrm{PuO}_{2}\right.$ pellets described in Figure 5b) good agreement is still observed for neutron doubles rates over a range of plutonium mass (shown in Figure $7 \mathrm{~b}$ ), the measurement result of primary concern for the design process. This 'miniature' system had promising ${ }^{252} \mathrm{Cf}$ absolute efficiencies of $4 \%$ for neutron singles and $0.06 \%$ for neutron doubles. This is good considering the final design will include many more detectors, covering a large solid angle. 

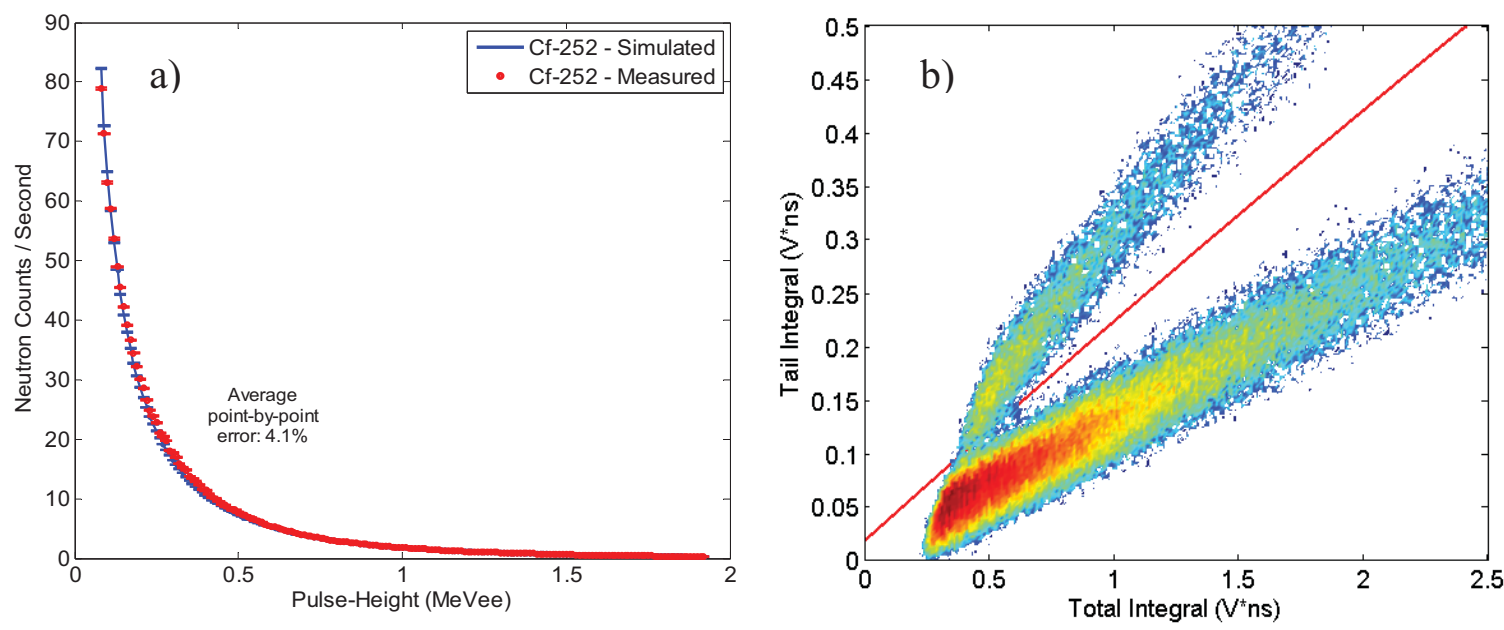

Figure 6 Simulated and measured ${ }^{255} \mathrm{Cf}$ pulse-height distributions (a) with an average point-by-point agreement error of $4.1 \%$. Photon discrimination (b) through PSD techniques at 70 keVee light-output threshold (approximately $650 \mathrm{keV}$ neutron energy deposited).
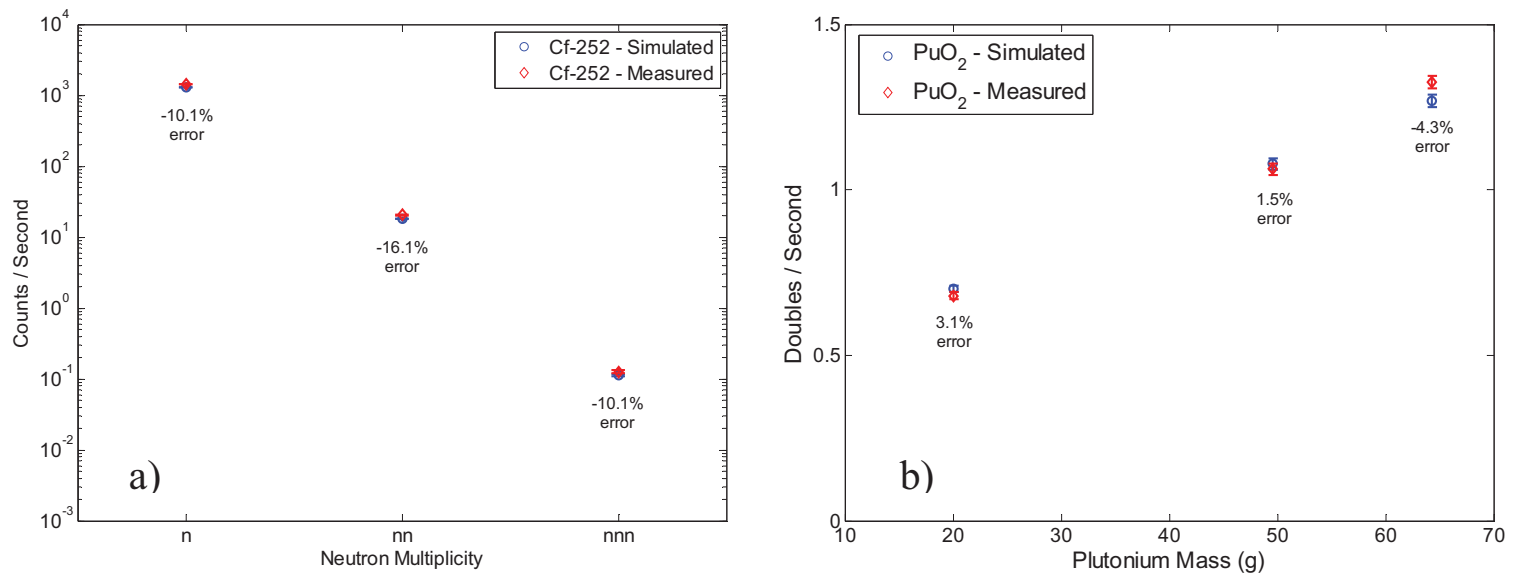

Figure 7 Simulated and measured ${ }^{252} \mathrm{Cf}$ neutron multiplicity values (a) with agreement errors between 10 and $16 \%$. Simulated and measured neutron doubles (b) from three measurements of $\mathrm{PuO}_{2}$ pellets of increasing mass with statistical errors between 2 and $4 \%$.

\subsubsection{Sensitivity of a Fast-Neutron Multiplicity Counter}

The plutonium-mass sensitivity of the measurement system was studied via the measured singles and doubles rates for $\mathrm{PuO}_{2}$ samples, shown in Figure 8. These preliminary results bode well for a full system's ability to quantify plutonium mass from fast-neutron multiplicity. Pulse-height information can classify the type of neutron source, as shown in Figure 9. This information can prove useful to tailor mass quantification equations to specific nuclear material types. 


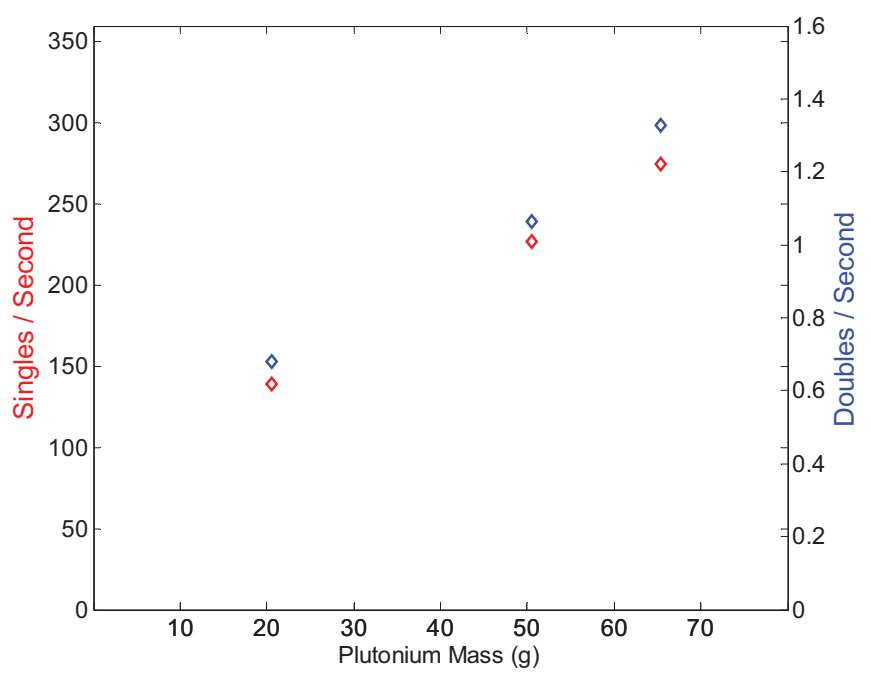

Figure 8 The relationship between neutron singles and doubles with plutonium mass, measured with the partial UM measurement system.

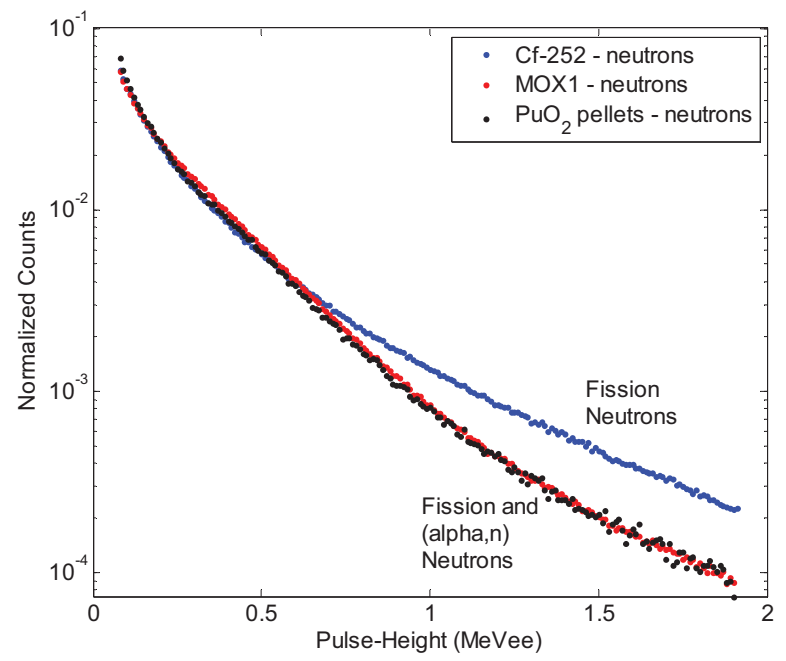

Figure 9 Measured neutron pulse-height distributions (normalized to their integral) for ${ }^{252} \mathrm{Cf}$, mixed-oxide powder (MOX), and the $\mathrm{PuO}_{2}$ pellets.

\subsubsection{Comparing Direct MCNPX PTRAC Data with MCNPX-PoliMi}

A comparison of a simulated data set produced with a standard version of MCNPX, using PTRAC file data, with the results of MCNPX-PoliMi, is shown in Figure 10. The standard MCNPX PTRAC file was processed to produce a list-mode file, with an additional analysis stage using a custom program for coincidence counting. The simulation used two cylindrical castings comprised of $93 \% \mathrm{HEU}$, with four plastic scintillators surrounding them. A ${ }^{252} \mathrm{Cf}$ spontaneous fission source was located in one casting. This geometrical arrangement and selection of materials was chosen to allow for direct comparison of the PTRAC reader and coincidence counting results with similar 
experimental measurements performed by Oak Ridge National Laboratory. Additional details regarding the measurements at ORNL are provided in reference 18 . For the standard MCNPX case, only neutrons with energies exceeding $1 \mathrm{MeV}$ and producing collisions within the scintillator volumes were processed. The secondary program extracted from the generated list-mode file all possible neutron pairs (between detectors 1 and 3) with "detection" times separated by 100 ns or less. The MCNPX-PoliMi model used an identical geometry, but also incorporated a built-in ${ }^{252} \mathrm{Cf}$ spontaneous fission source distribution. The detector output file was then processed with the accompanying MPPost software, which produced cross correlations between detectors 1 and 3 with an accepted $\Delta \mathrm{t}$ of $\pm 100 \mathrm{~ns}$. Both simulation methods utilized bin widths of $2 \mathrm{~ns}$. There is strong agreement between the two methods over the complete range of $\Delta \mathrm{t}$ 's. Certainly, this result is somewhat expected since both methods are essentially recording data on a "per track" basis. The principle advantage of possessing custom software to read the PTRAC file and process the information is simply the ability to customize or tailor the process and outputs. The combination of MCNPX-PoliMi and MPPost has its own distinct advantages for this type of modeling and analysis, particularly when attempting to produce more realistic detector characteristics (e.g., scintillator light output).
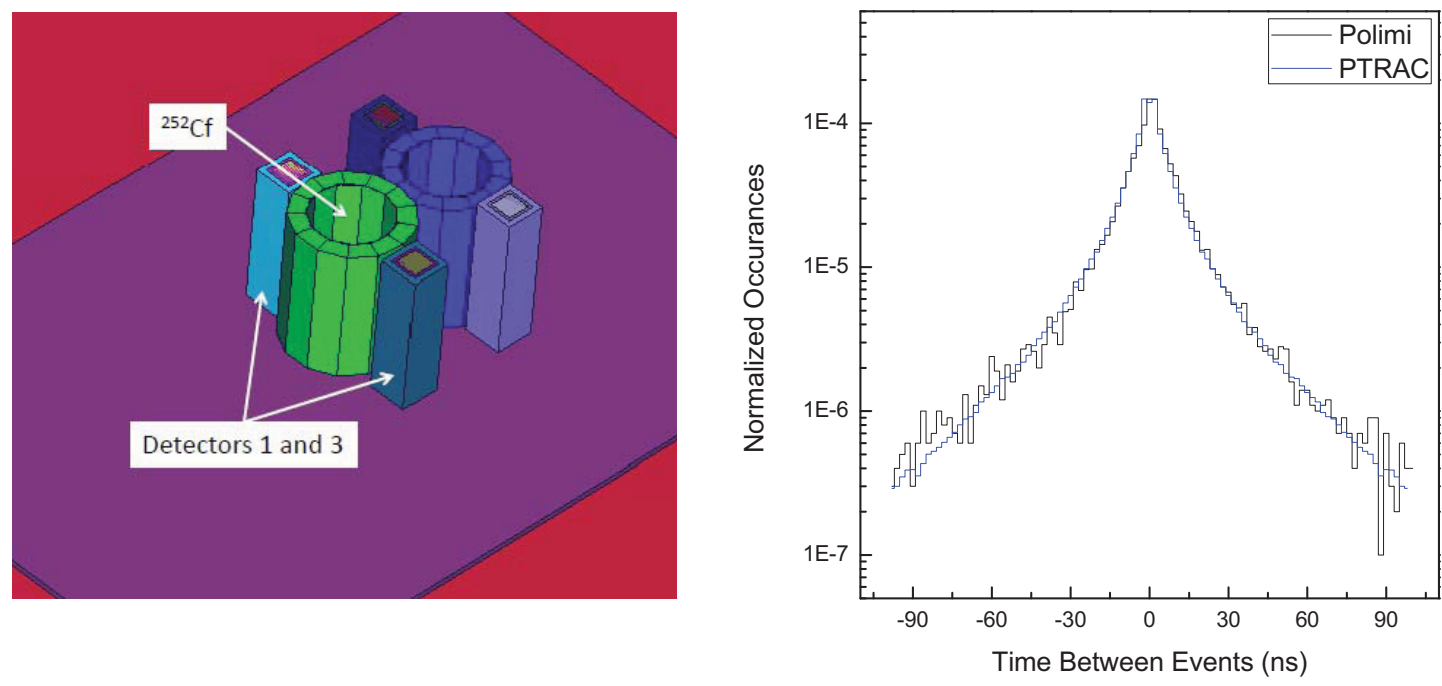

Figure 10 Left: geometry setup for these simulations. The two cylinders are $\sim 93 \%$ HEU castings, with a ${ }^{252} \mathrm{Cf}$ spontaneous fission source in the middle of the left casting. Cross correlations were simulated between detectors 1 and 3 . Right: comparison of the output of a custom PTRAC reader and coincidence counting program with output from MCNPX-PoliMi.

\subsection{Conceptual System Layout}

Current simulation efforts focus on studying trends in the detector shape, size, number, and configuration to achieve high efficiency, high sensitivity, and minimal dependence on sample placement; examples are shown in Figure 11. An ideal detector design will not only perform accurately and efficiently, but also maintain a minimally intrusive geometry in terms of size and weight. With a list of candidate designs, bench top experiments are being performed at UM to work towards fine-tuning the design. 
a)

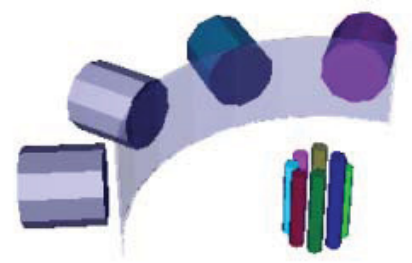

c)

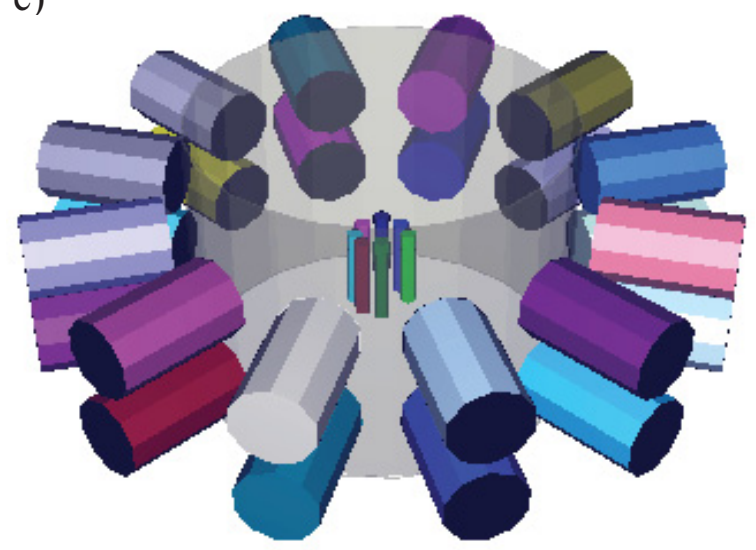

b)

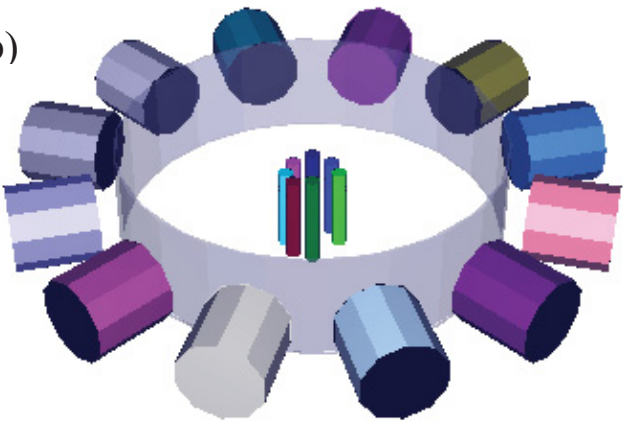

d)

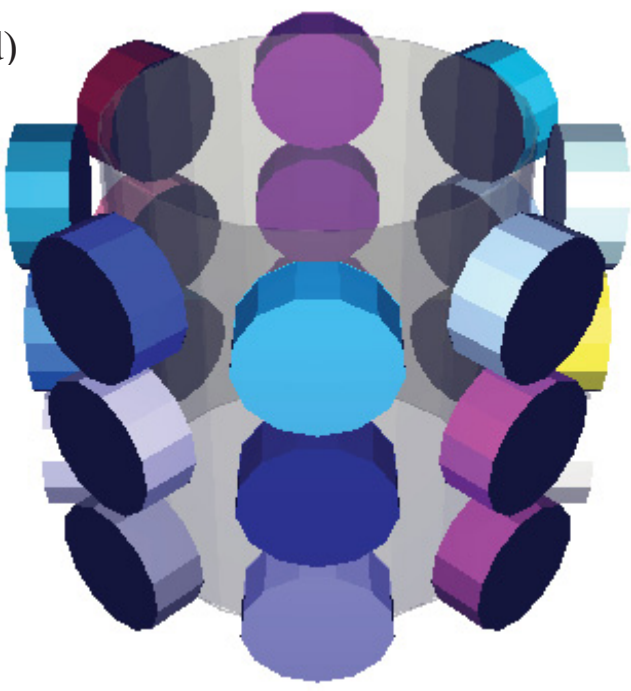

Figure 11 Example of fast-neutron, multiplicity-counter models (a) JRC measurement system based on EJ-309 liquid scintillators, (b) a full ring (12 detectors) of 3" $\times 3$ 3" EJ-309 liquid scintillators, (c) two rings of 3" $\times 5$ 5" EJ-309 liquid scintillators, and (d) three rings of 5" $\times 2$ " EJ-309 liquid scintillators. The models include the active volume of the liquid scintillators, 0.1 " of lead shielding, and $\mathrm{PuO}_{2}$ pellets.

\subsection{Design Studies Discussion}

Using MCNPX-PoliMi/MPPost many detector configurations were tested with numerous types of plutonium-containing materials of varying plutonium mass. The results shown below focus on how a system would respond to the JRC's $\mathrm{PuO}_{2}$ pellets as they were measured during the campaign described above (detectors remain $20 \mathrm{~cm}$ from the measured sample, 0.1 inches of lead shielding was present, and a $70 \mathrm{keVee}$ threshold light-output threshold (approximately $650 \mathrm{keV}$ neutron energy deposited was applied)). Simulated results in Figure 12 show how the doubles rate, from various system designs, trends with increasing plutonium mass. Designs included either one, two, or three rings of liquid scintillators. The liquid scintillator dimensions were either 3 or 5 inches in diameter and varied in length from 1 to 5 inches. The slope of the doubles rate curves 
shown in Figure 12 is then the sensitivity metric for determining the responsiveness of the simulated systems to plutonium mass. The sensitivity of each of the depicted designs is given in Table 1. Systems that subtend the most solid angle and have the greatest detector volumes perform best, as anticipated.
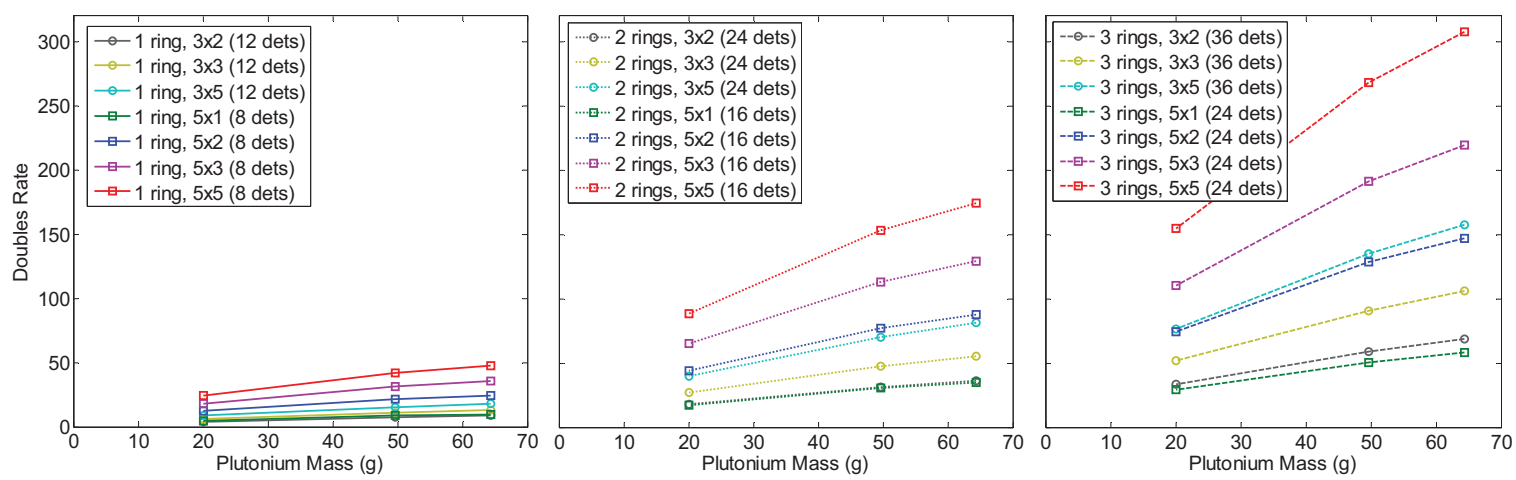

Figure 12 The trend of simulated doubles rates with plutonium mass for 21 fastneutron multiplicity counter designs. The number of detectors and the detector size were both varied. The figures compare the response according to the number of detector rings.

Table 1 The doubles-rate sensitivity for the designs presented in Figure 12.

\begin{tabular}{|c|c|c|c|}
\hline \multirow{2}{*}{$\begin{array}{l}\text { Detector Shape } \\
\text { (diameter } x \\
\text { length) }\end{array}$} & \multicolumn{3}{|c|}{$\begin{array}{c}\text { Sensitivity } \\
\text { (Doubles Rate / g of plutonium) }\end{array}$} \\
\hline & One Ring & Two Rings & Three Rings \\
\hline $3 "$ x 2" & 0.10 & 0.42 & 0.81 \\
\hline 3" x 3" & 0.15 & 0.65 & 1.25 \\
\hline $3^{\prime \prime}$ x 5" & 0.22 & 0.96 & 1.85 \\
\hline 5" × 1" & 0.11 & 0.40 & 0.67 \\
\hline $5 " \times 2 "$ & 0.28 & 1.00 & 1.68 \\
\hline $5 "$ × 3" & 0.41 & 1.47 & 2.50 \\
\hline 5" x 5" & 0.55 & 1.98 & 3.51 \\
\hline
\end{tabular}

\section{CONCEPTUAL DESIGN}

A design, such as 3 rings of $5 " \times 5$ " detectors, performs best but may be too cumbersome in size. Therefore a more appropriate choice may be a system more similar to the 2 rings of 5 " $\times 3$ " length detectors, which does not take a significant hit in sensitivity while maintaining more compact system dimensions. Additionally, when we consider UM's current data acquisition capabilities for large numbers of digitized channels, the system's design will be limited primarily by data acquisition rates. At this 
point in time, in order to perform the necessary off-line data analysis, including PSD, most available systems require that the entire waveform for each detection be transferred to a computer. Using an optical link, the maximum available data transfer rate is $\sim 76$ $\mathrm{MB} / \mathrm{s}$ and each waveform is on the order of 200 bits. With the high expected count rates associated with advanced nuclear fuels, there will be a limit to the allowable efficiency of the system. When considering approximately $8 \mathrm{~kg}$ of MOX, these limitations eliminate the most sensitive designs, such as three rings of 5" diameter detectors, and even nearly eliminate some of the two ring designs with 5" diameter detectors.

Ongoing efforts and advances in data acquisition hardware and software can work towards reducing this constraint. Systems that have recently become available on the market are significantly better in terms of data acquisition and data transfer capabilities. Such systems will also allow the UM data analysis to be implemented on-the-fly. The next year of this research project will address integrating these advanced systems into the UM measurement system.

\subsection{Expected Performance -2 Rings of $5 " \varnothing \times 3$ " Detectors}

To highlight the potential performance of a liquid scintillator fast neutron multiplicity counter, the focus is placed on the 2 rings of 5 " $\times 3$ " detectors. This system's expected plutonium mass values, and the mass uncertainties for the simulated doubles of 1-minute measurements of the $\mathrm{PuO}_{2}$ masses, are shown in Figure 13.

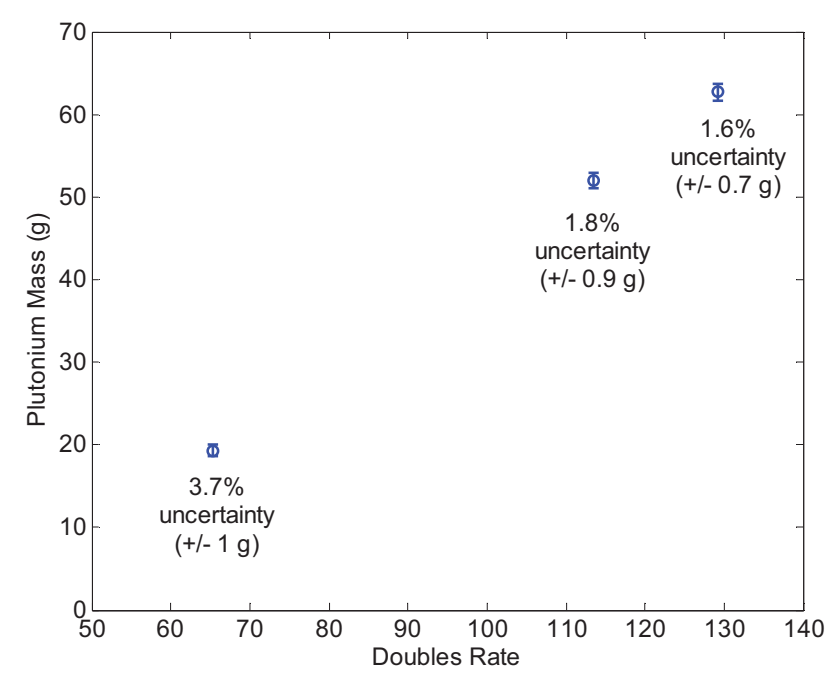

Figure 13 Plutonium mass statistical uncertainty for a 1-minute measurement with the two rings of 5 " $\times 3$ " length design.

For small plutonium mass samples, this is a very promising measurement time for such reasonable accuracy. The mass uncertainty will decrease with measurement time for each plutonium mass, as shown in Fig. 10. This supports the claim that fast-neutron multiplicity systems can be efficient enough to give accurate results quickly for even small amounts of plutonium. It is important to note that these mass uncertainties are based on statistical uncertainty only; error produced due to PSD misclassification can 
potentially create very large mass uncertainty. It will be of utmost importance to ensure excellent PSD to maintain such low uncertainty.

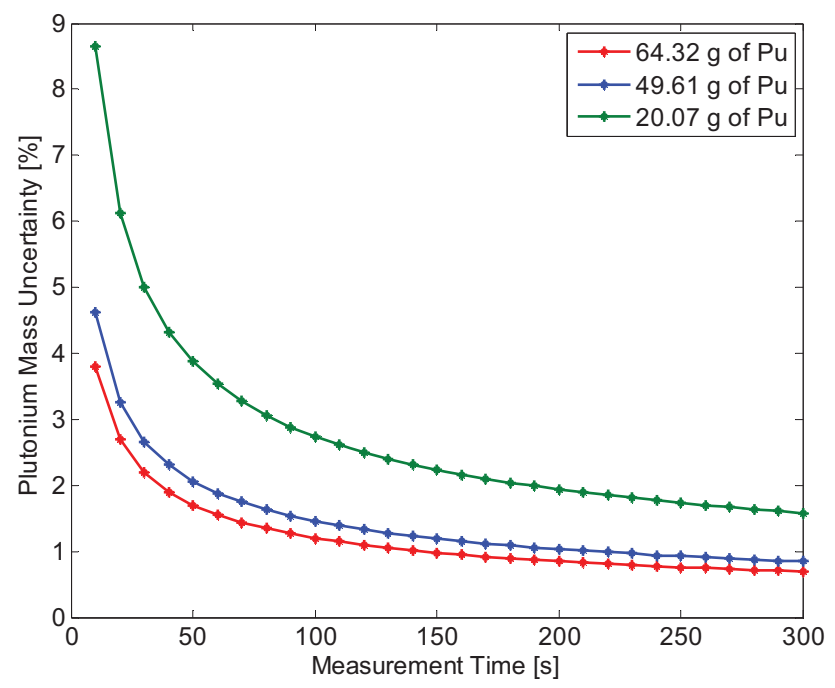

Figure 14 Plutonium mass uncertainty as a function of measurement time, for the three $\mathrm{PuO}_{2}$ pellet cases simulated with the two rings of $5 " \times 3$ " length design.

\subsection{Future Advances Applicable to the Design}

With the MCNPX-PoliMi simulation efforts, conceptual designs have been studied and the conclusion can be made that more detectors and greater total detector volume will result in a more sensitive system. Settling on a final configuration will then rely on limits of the electronics. Fortunately, a significant amount of state-of-the-art research is currently taking place to push the limits of current data acquisition hardware and software. The next stage of research will be focused on laboratory testing of a variety of detector configurations with data acquisition advances to finally narrow down a design for prototyping. Data acquisition system development efforts are summarized below.

System High-Voltage Supply and Auto-Calibration - The MPod mini crate (WIE-NE-R, Plein \& Baus Corp.) is capable of providing high voltage to arrays containing many detectors. The hardware is capable of producing a maximum voltage of $3000 \mathrm{~V}$ and a maximum current of $1.5 \mathrm{~mA}$. In our work, the MPod currently contains three 16channel boards (Figure 15) that are controlled by accessing the system though a local area network (LAN). This feature allows for creation of software that can automatically calibrate large detector-arrays. Once a measurement is taken with a calibration source, the program will automatically adjust the bias to the detector until a desirable gain is reached. The remote adjustment feature of the MPod eliminates the need for the system operator to manually adjust the voltage bias allowing for rapid calibration. This software will also include monitoring for any inherent gain shifts that may occur in a photomultiplier tube and take action to recalibrate the detector on the fly. Current UM efforts focus on finalizing and testing the automated calibration and gain control software. 


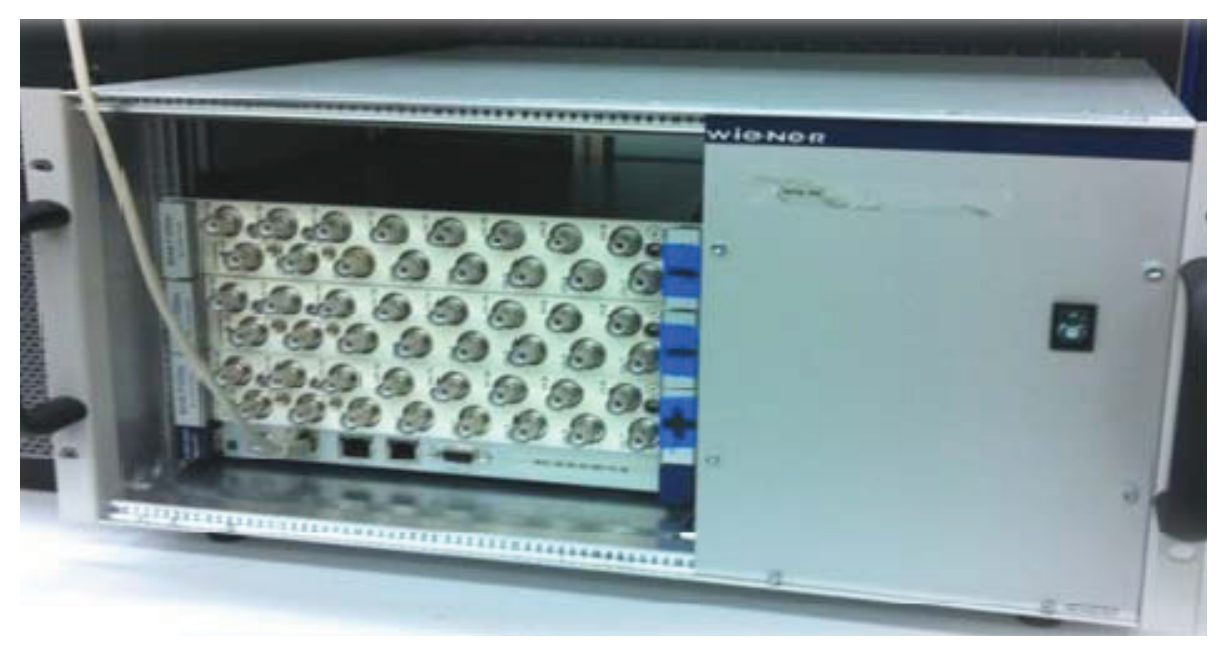

Figure 15 Three, LAN controllable, 16-channel high-voltage boards contained in an MPod mini-crate for supplying a bias to a large quantity of detectors.

Digitizer Board Synchronization - The current conceptual designs revolve around the idea of digitized waveforms for accurate data analysis including subnanosecond timing and detailed PSD. To accommodate a full fast neutron multiplicity counter, current available digitizer technology requires that separate digitizer boards with a limited number of channels are paired together to digitize signals from all liquid scintillators. The current UM data acquisition system uses 8-channel CAEN V1720 digitizers. Therefore, a system utilizing between 16 and 36 detectors would require between 2 to 5 separate V1720 digitizers (shown in Figure 16). In order to maintain subnanosecond timing for all channels across all of the digitizers, the boards must be accurately synchronized in time. This crucial step has been achieved for the UM measurement system and is currently under further development to maximize system count rate limits. Other research efforts are focused on developing field programmable gate arrays that are programmed to do on-the-fly data analysis but are currently limited to four channels.

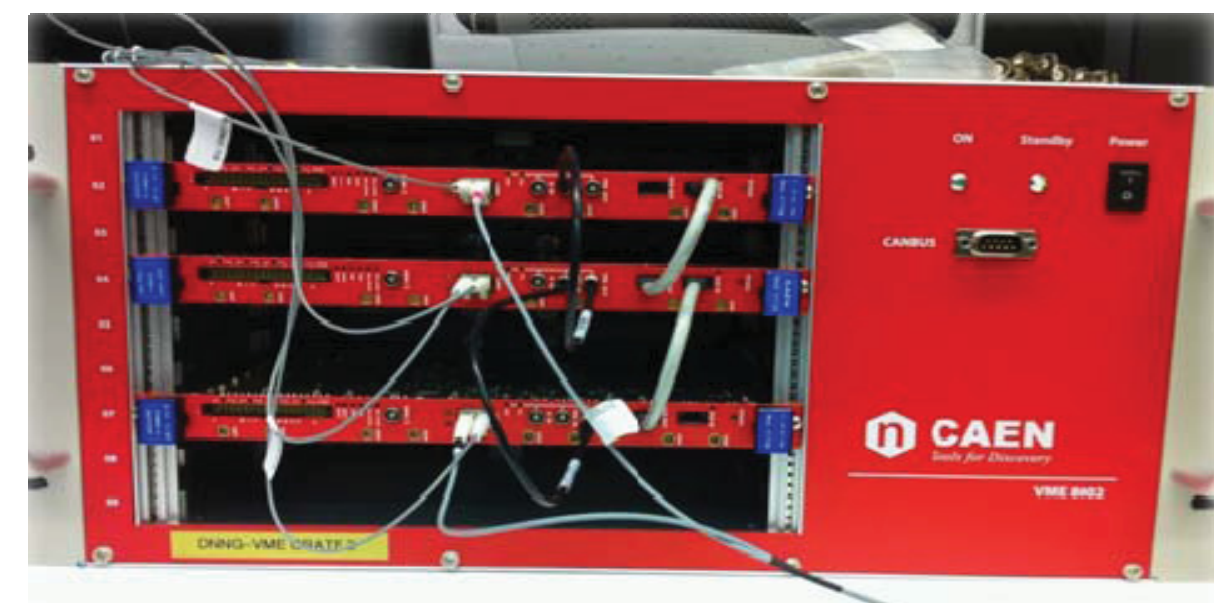

Figure 16 Three, time-synchronized CAEN V1720 digitizers (encompassing 24 detector channels) where information is passed via optical links. 
Digitizer Hardware and Firmware Advancements - Data analysis and mass determination algorithms will be cable of being performed on-the-fly with improvements in the size of FPGA's available in multi-channel digitizers. UM has currently implemented a number of on-the-fly capabilities on a four-channel digitizer (X5-210M from Innovative Integration): pulse-height identification, accurate timing determination, and PSD. Recently available digitizers and digitizer crates will allow all algorithms to be stored on the digitizer and time synchronization between digitizers to be inherent. With new computer connectivity abilities, such as USB 3.0, data transfer capabilities will increase by potentially a factor of five. When combining on-the-fly data analysis and improved connectivity, count-rate limitations will no longer be an issue as the measurement system will process all relevant information directly on the digitizer's board and will provide the quantities of interest. Investigation has already been performed on the best fits for this application and the next phase of this research project will include inlab testing of these new technologies for the application of fast neutron detection with liquid scintillators.
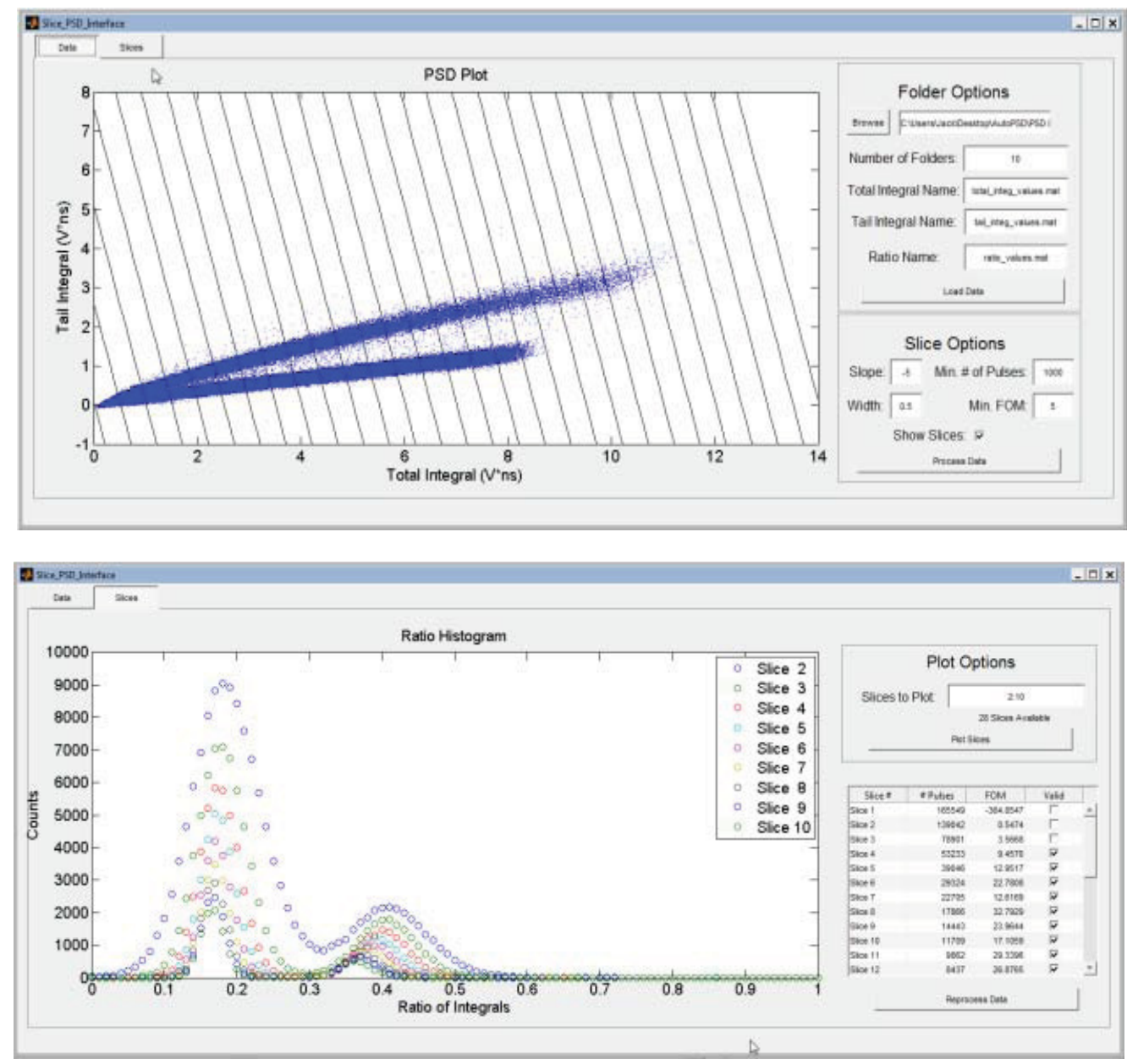

Figure 17 A pulse-shape discrimination method that can be automated to perform more accurate photon and neutron discrimination across the entire range of pulse heights measured in the liquid scintillators. 
Pulse-Shape Discrimination - To successfully employ a fast neutron multiplicity counter, it is of utmost importance that we can accurately apply PSD to distinguish neutron detections from photon detections in the liquid scintillators. When measuring advanced fuel cycle materials with high photon to neutron ratios, the photon misclassification error will be magnified. Current UM PSD algorithms result in approximately a $1 / 1000$ photon misclassification rate at very low thresholds of $\sim 460 \mathrm{keV}$ deposited neutron energy ( $\sim 40 \mathrm{keVee}$ ). UM research efforts are working to improve this rate with hybrid PSD methods, applying various algorithms to numerous categories of waveforms in certain pulse-height ranges. Additional efforts include an automated PSD software that finds the best photon/neutron discrimination line as the data evolves. An illustration showing how the PSD software seeks the most accurate photon/neutron discrimination across the measured range of energies is presented in Figure 17.

Alternative Data Acquisition Hardware - At INL work is planned to begin assessing the utility of a digitizer architecture based on new a product from Struck Innovative Systeme. Struck digitizer systems have been used at several different U.S. national laboratories for operating arrays of liquid-scintillator detectors for several years, including Oak Ridge National Laboratory (ORNL) and Lawrence Livermore National Laboratory.[11] In FY2012 INL begin working with an eight channel Struck digitizer card on loan from ORNL (courtesy of Paul Hausladen and Jason Newby), see Figure 18. Using this card as a learning tool, new control and data acquisition software is being developed at INL to support on-line, multi-board synchronized data acquisition using liquid scintillators. In FY2013 this work will escalate, following the arrival of a newly purchased, 16-channel SIS3316-250-14 Struck digitizer card (250 MHz, 14-bit).

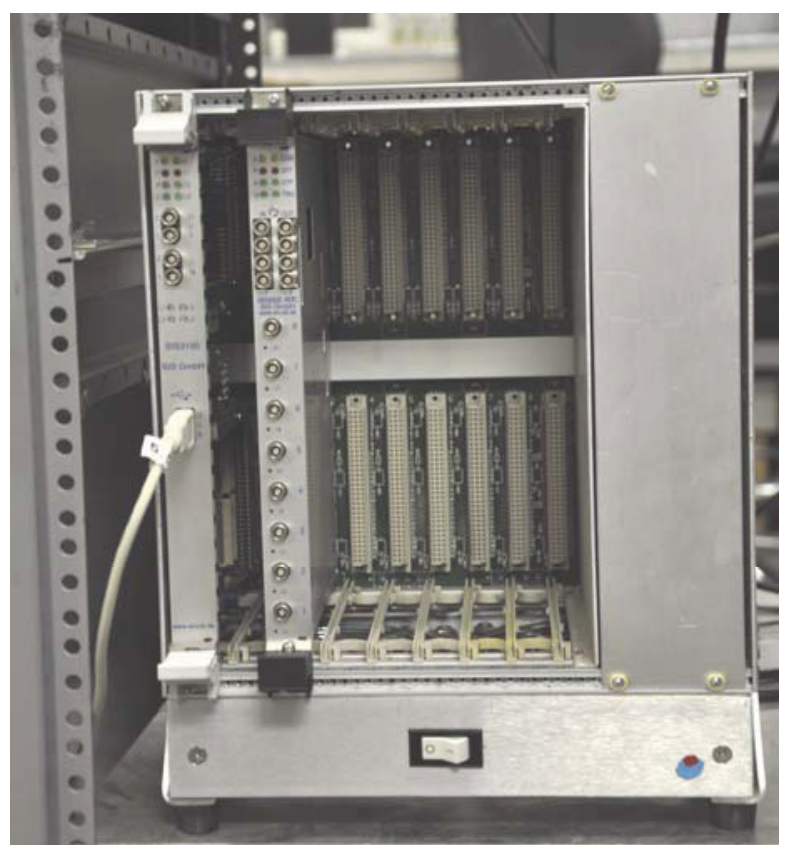

Figure 18 An eight-channel Struck SIS3302 8-channel digitizer card under evaluation at INL. 
Evaluation of Non-Standard Detector Geometry - Although the majority of all PSD-capable organic scintillator detectors (either liquid or plastic) are cylindrical in shape this form-factor is not a prerequisite for successful pulse-shape discrimination. For example, recent work in the INL-UM collaboration has evaluated and compared the performance of comparably-sized square-shaped liquid-scintillator detector modules with cylindrical modules. The PSD-performance of the non-cylindrical detectors is somewhat degraded in comparison with the cylindrical detectors but is still sufficient for most neutron-measurement applications. An advantage of non-cylindrical detector packages is that designs may be more easily arranged into a well-like measurement geometry of the type most often used for safeguards Pu-assay work. In concept, a spherical-cavity could be used but, since most items to be analyzed are packaged in cylindrical containers, a cylindrical-shaped sample area is most optimal for this application. Recognizing this, it is possible that pie-shaped wedges might be the most optimal layout, for example. Prior work has examined the use of long, cylindrical-tube geometry for these detectors.[19] Unfortunately, this layout leads to unusually poor PSD performance and is often not optimal for this application. Work is planned to continue studying the trade-offs between shape and PSD-performance for Pu-assay applications.

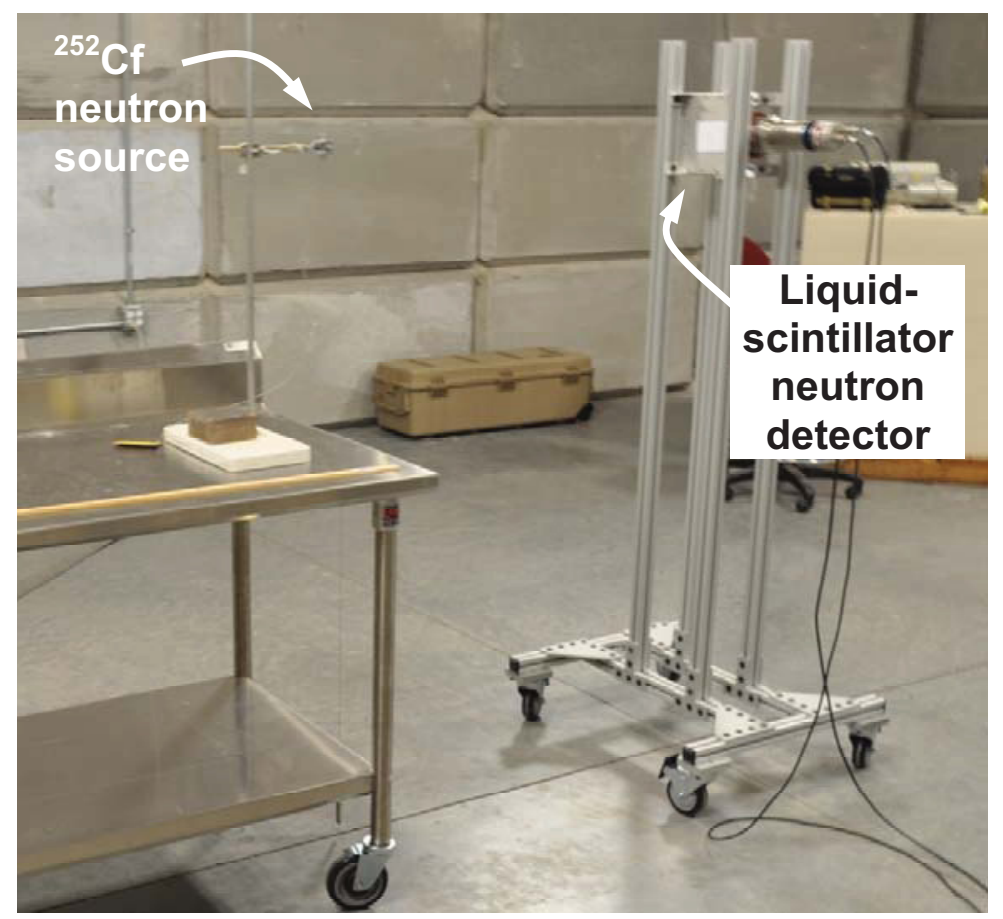

Figure 19 Test activities at INL to evaluate the performance of a square-shaped liquid-scintillator filled with EJ-309 material.

\subsection{Experimental Next Steps}

Ongoing efforts to improve the final conceptual design include: characterizing cross-talk with bench top measurements using liquid scintillators at INL and UM, testing data-analysis algorithms to minimize cross-talk contribution to measured doubles and triples, optimization of detector placement relative to other detectors, and simulations of 
the potential benefit of additional materials placed within the system. Novel PSD capable plastic organic scintillation detectors are being tested with the UM measurement system to assess if the PSD performance is adequate for consideration in a fast neutron multiplicity counter. Plastic scintillators eliminate some of the concerns about the fieldability of liquid scintillators. Photomultiplier tubes and plastic scintillators have proven to be rugged in applications such as oil-well logging. Novel detector materials or innovation in electronics such as photomultiplier tube replacement, field programmable gate arrays, or digitizers can be applied to an optimized fast neutron multiplicity counter design.

For development of the data-analysis algorithms, future measurements at INL with an expanded list of fissile materials (Fig. 14) will help to characterize a more final system design. By studying the system response to a wider range of SNM, the mathematics to accurately quantify fissile material can be enhanced.

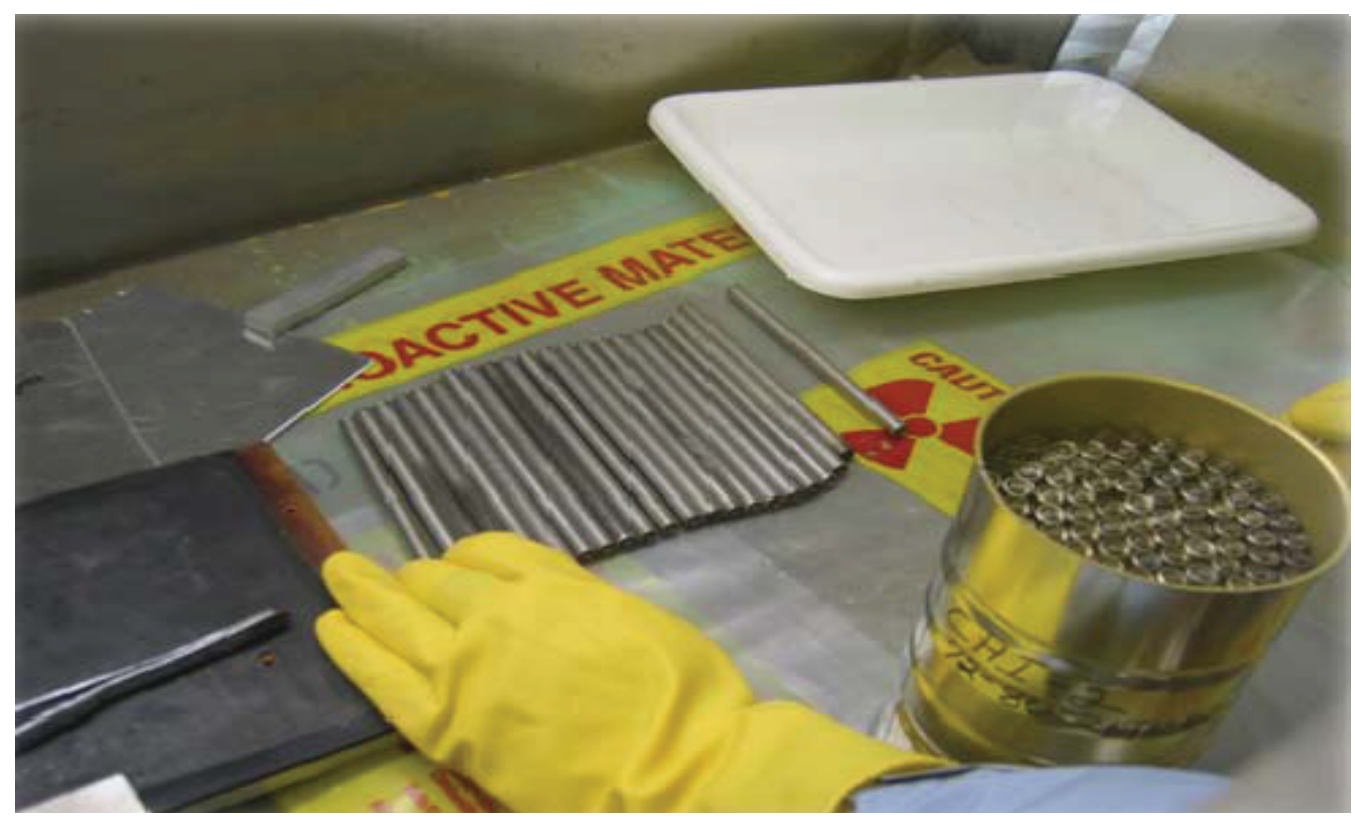

Figure 20 MOX fuel pins available for measurement and fast neutron multiplicity counter calibration at INL.

\section{SUMMARY}

Studies have been performed to assess the conceptual performance capabilities of a fast-neutron multiplicity counter for assaying plutonium. Comparisons have been made to evaluate the potential improvements and benefits of fast-neutron multiplicity analyses versus traditional thermal-neutron counting systems. Fast-neutron instrumentation, using for example an array of liquid scintillators such as EJ-309, have the potential to either a) significantly reduce assay measurement times versus traditional approaches, for comparable measurement precision values, b) significantly improve assay precision values, for measurement durations comparable to current-generation technology, or c) moderately improve both measurement precision and measurement durations versus current-generation technology. Using the MCNPX-PoliMi Monte Carlo simulation code, 
studies have been performed to assess the doubles-detection efficiency for a variety of counter layouts of cylindrical liquid scintillator detector cells over one, two, and three rows.

Ignoring other considerations, the best detector design is the one with the most detecting volume. However, operational limitations guide a) the maximum acceptable size of each detector cell (due to PSD performance and maximum-acceptable per-channel data throughput rates, limited by pulse pile-up and the processing rate of the electronics components of the system) and b) the affordability of a system due to the number of total channels of data to be collected and processed. As a first estimate, it appears that a system comprised of two rows of detectors $5 " \varnothing \times 3$ " would yield a working prototype system with excellent performance capabilities for assaying Pu-containing items and capable of handling high signal rates likely when measuring items with $\mathrm{Pu}$ and other actinides. However, it is still likely that gamma-ray shielding will be needed to reduce the total signal rate in the detectors. As a first step prior to working with these largersized detectors, it may be practical to perform scoping studies using small detectors, such as already-on-hand $3 " \varnothing \times 3 "$ detectors. In parallel with future test and benchmarking activities, it will also be important to continue working to improve the modeling fidelity for this project. Of particular impatience will be a focus on the assessing and modeling the gamma-ray component of the source term for these types of objects. Lastly, continued work is needed towards the optimization and automation of a comprehensive data acquisition system to support fast-neutron multiplicity detection using liquid scintillations. Prior work has shown that operational issues, including long-term stability of the electronics for these systems, can play a deleterious role in their performance for conducting Pu-assay measurements.[11] 


\section{REFERENCES}

1 Chichester, D. L., et al., "FY09 Advanced Instrumentation and Active Interrogation Research for Safeguards," Report INL/EXT-09-16611, Idaho National Laboratory, Idaho Falls, Id. (2009).

2 Chichester, D. L., et al., "MPACT FY2011 Advanced Time Correlated Measurement Research at INL," Report INL/MIS-11-23379, Idaho National Laboratory, Idaho Falls, Id. (2011)

3 ne.doe.gov (2005) DOE - Office of Nuclear Energy. [online] Available at: http://www.ne.doe.gov/AFCI/neAFCI.html [Accessed: Aug 2012].

4 un.org (1968) UNODA - Non-Proliferation of Nuclear Weapons (NPT). [online] Available at: http://www.un.org/disarmament/WMD/Nuclear/NPT.shtml [Accessed: Aug 2012].

5 Ensslin, N., et al., "Application Guide to Neutron Multiplicity Counting," Report LA-13422-M, Los Alamos National Laboratory, Los Alamos, N.M. (1998).

6 www.canberra.com.

7 Canberra.com (2000) Neutron Safeguards Systems - CANBERRA Industries. [online] Available at: http://www.canberra.com/products/waste_safeguard_systems/ neutron-safeguards-systems.asp [Accessed: Sept 2012].

8 Pázsit, I., Enqvist, A., and Pál, L., "A Note on the Multiplicity Expressions in Nuclear Safeguards," Nucl. Inst. Meth. Phys. Res. A 603 (2009) 541-544.

9 Dytlewski, N., Krick, M. S., and Ensslin, N., "Measurement Variances in Thermal Neutron Coincidence Counters," Nucl. Inst. Meth. Phys. Res. A 290 (1990) 197-207.

10 Reilly, D., Ensslin, N., and Smith, H., (eds.), "Passive Nondestructive Assay of Nuclear Materials," Report NUREG/CR-5550, U. S. Nuclear Regulatory Commission, Washington, D. C. (1991 and 2007).

11 Henzlova, D., "Epithermal Neutron Multiplicity Counter (ENMC) Measurement Description and Results," Report LA-UR-11-00094, Los Alamos National Laboratory, Los Alamos, N.M. (2010).

12 McConchie, S., Hausladen, P., and Mihalczo, J., "Prompt Neutron Decay Constant from Feynman Variance Fitting," Oak Ridge National Lavatory, Oak Ridge, Tenn. (2010). (Unpublished)

13 Miller, M. C., et al., "Design of a Fast Neutron Coincidence Counter," App. Rad. Iso. 48 (1997) 1549-1555.

14 Ensslin, N., Dytlewski, N., and Krick, M. S., "Assay Variance as a Figure of Merit for Neutron Multiplicity Counters," Nucl. Inst. Meth. Phys. Res. A 290 (1990) 197-207.

15 Padovani, E., et al., "Introduction to MCNPX-PoliMi", Version 2.7, University of Michigan, Ann Arbor, Mich. (2012).

16 Miller, E. C., et al., "MCNPX-PoliMi Post-Processor (MPPost) Manual," Version 2.1.0, University of Michigan, Ann Arbor, Mich. (2012).

17 Pozzi, S. A., "MCNPX-PoliMi for Nuclear Nonproliferation Applications," Nucl. Inst. Meth. Phys. Res., 694 (2012) 119-125.

18 Mihalczo, J., "Subcriticality Measurements with HEU (93.2) Metal Annular Storage Castings," Report ORNL/TM-2007/134, Oak Ridge National Laboratory, Oak Ridge, Tenn. (2007).

19 Frame, K., et al., Development of a Liquid Scintillator Neutron Multiplicity Counter (LSMC)," Nucl. Inst. Meth. Phys. Res. A 579 (192-195. 


\section{APPENDIX}

This appendix contains the slides presented at the MPACT end-of-year meeting in Idaho Falls, Id., on August 29, 2012 (INL/MIS-12-27008). 

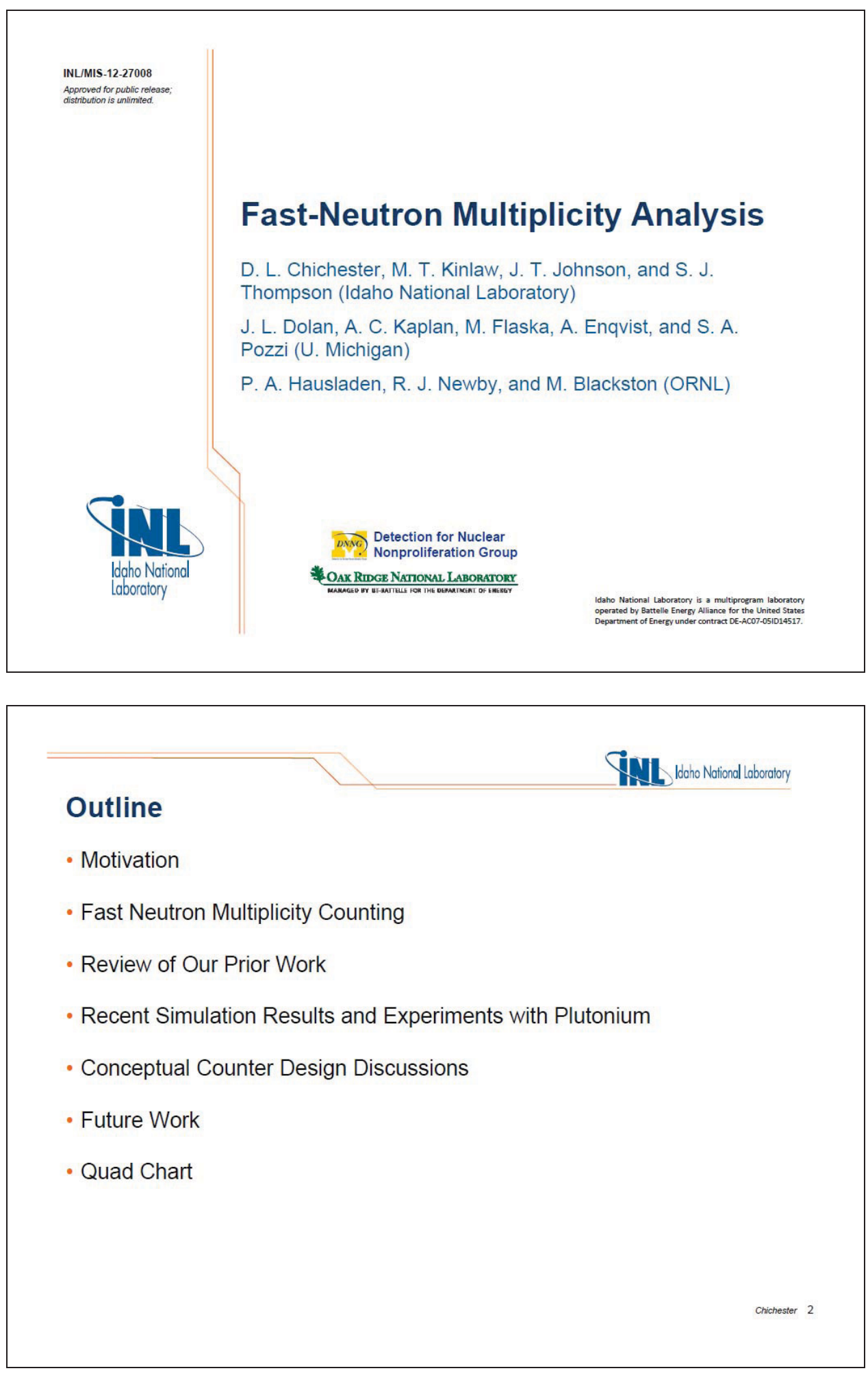


\section{Fuel Cycle Technologies (FCT) Program}

- The FCT program is charged with developing used fuel and waste management strategies and sustainable fuel cycle options that improve resource utilization, responsibly manage wastes, improve safety, and limit proliferation risk.

Sustainable nuclear fuel cycle options are those that improve uranium resource availability and utilization, minimize waste generation, and provide adequate capability and capacity to manage all wastes produced by the nuclear fuel cycle.

- The Advanced Fuels Campaign is currently studying fuels that can transmute longlived transuranic isotopes contained in UNF into shorter-lived fission products.

\begin{tabular}{|c|c|c|c|c|}
\hline Test Series & $\mathbf{U}, \%$ & $\mathbf{P u}, \%$ & $\mathbf{N p}, \%$ & Am, $\%$ \\
\hline AFC-1D (metallic) & 0 & $70-100$ & $0-15.24$ & $0-16$ \\
\hline AFC-1G (nitride) & $0 / 50$ & $50 / 25$ & $9-25$ & $15-50$ \\
\hline AFC-1H (metallic) & 50 & 41 & 2.5 & 6.1 \\
\hline AFC-2A (metallic) & 70 & 24 & 2.4 & 3.6 \\
\hline AFC-2B (metallic) & 52 & 38 & 3.8 & 6.3 \\
\hline
\end{tabular}

- TRU compositions are based on the expected TRU content by group extraction from recycled spent fuel from light water reactors with an average burnup of 50 $\mathrm{MWd} / \mathrm{MT}$ and cooled for 40 years.

\section{Plutonium and Minor Actinides (MAs)}

- Work is underway to expand the use of uranium-plutonium mixed oxide (MOX) fuels in current-generation light water reactors, both domestically and internationally.

- Future fuel cycle strategies currently under development seek to combine plutonium along with other minor actinides for waste minimization and energyrecovery optimization.

- Future fuel reprocessing strategies currently under development seek to keep plutonium, minor actinides, and some fission products co-mingled to reduce proliferation risks.

- Assay methods capable of determining Pu in metallic and oxide Pu forms, and in $\mathrm{U} / \mathrm{Pu}$ and U/Pu/MA MOX fuels, are important now and will become more important in the future. Improvements are needed to improve the precision of these measurements, to reduce analysis times, and to avoid the use of helium-3. 


\section{Fast-Neutron Multiplicity Counting}

Idaho National Laboratory

- Fast-neutron counting has several potential advantages over the thermal and epithermal neutron counters that are currently used for the nondestructive assay of plutonium packages.

- Short die-away time, $\tau, \mathcal{O}(10 \mathrm{~ns})$ :

Allows assay of higher-order multiplicity with fewer random events.

Supports assays of samples with high $(\alpha, n)$ source term.

- Supports assays using active neutron interrogation sources.

- Reduces counting times to reach proscribed uncertainty levels.

Supports high-throughput operations.

- Fast response times:

- Allows analysis of high count rate materials.

- May allow use of prompt-fission-neutron anisotropy as a new analytical parameter.

- Pulse-height analysis:

- May allow use of neutron energy information (support new methods for active interrogation).

- Pulse-shape discrimination:

- May support use of neutron-photon coincidence analysis.

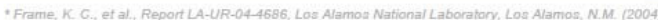

$\begin{gathered}\text { Relative Standard } \\ \text { Deviation, } R S D^{\star}\end{gathered}$
RSD $\propto \frac{\sqrt{\tau}}{\varepsilon \sqrt{t \mathrm{~m}}}$
$\tau=$ die-away time
$\varepsilon=$ efficiency
$t=$ counting time
$m={ }^{240} \mathrm{Pu}_{\mathrm{eff}}$ mass

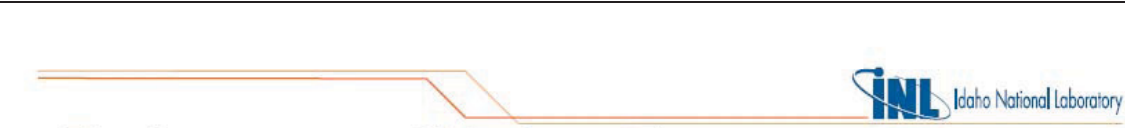

The Importance of Die-Away Time

Fast-Neutron Counting

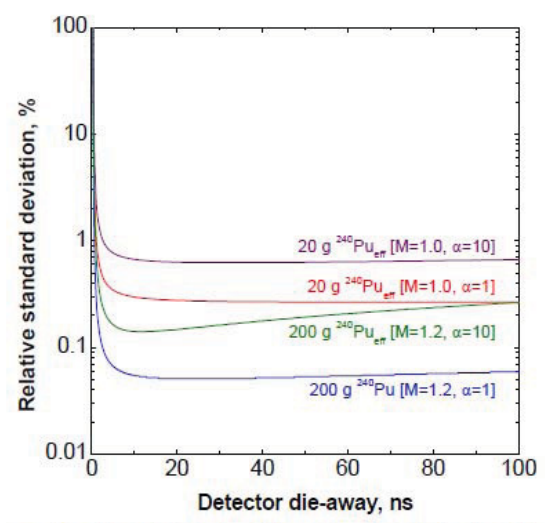

Slow-Neutron Counting

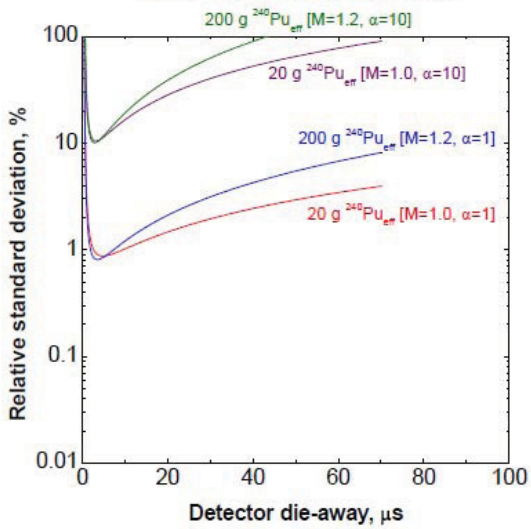

- Accidental coincidences are not significant for the fast-neutron counter.

- For thermal counting the high $(\alpha, n)$ rate significantly degrades RSD but for fast counting the RSD is mostly dominated by total rate and detector efficiency. 


\section{The Importance of Die-Away Time}
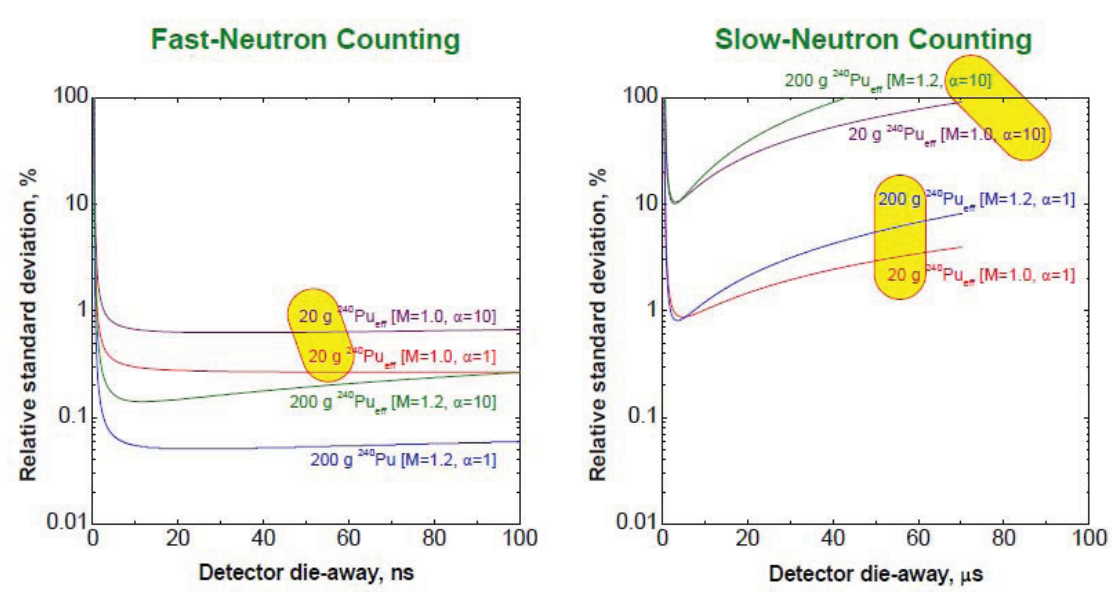

- Accidental coincidences are not significant for the fast-neutron counter.

- For thermal counting the high $(\alpha, n)$ rate significantly degrades RSD but for fast counting the RSD is mostly dominated by total rate and detector efficiency.

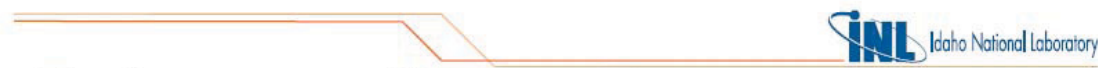

The Importance of Die-Away Time

Fast-Neutron Counting

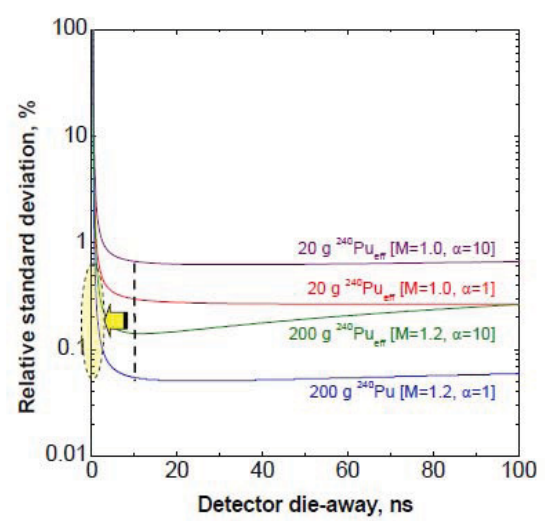

Slow-Neutron Counting

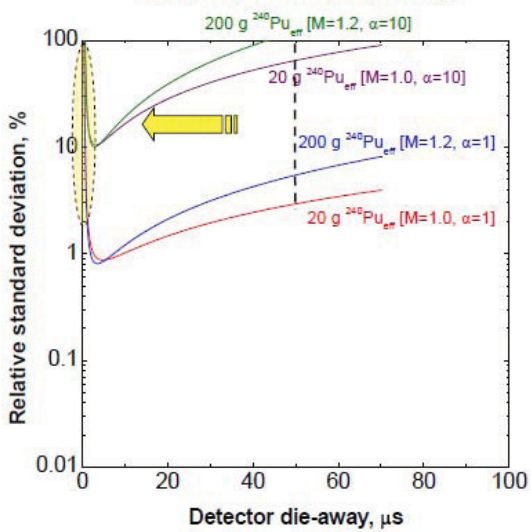

- Accidental coincidences are not significant for the fast-neutron counter.

- For thermal counting the high $(\alpha, n)$ rate significantly degrades RSD but for fast counting the RSD is mostly dominated by total rate and detector efficiency. 

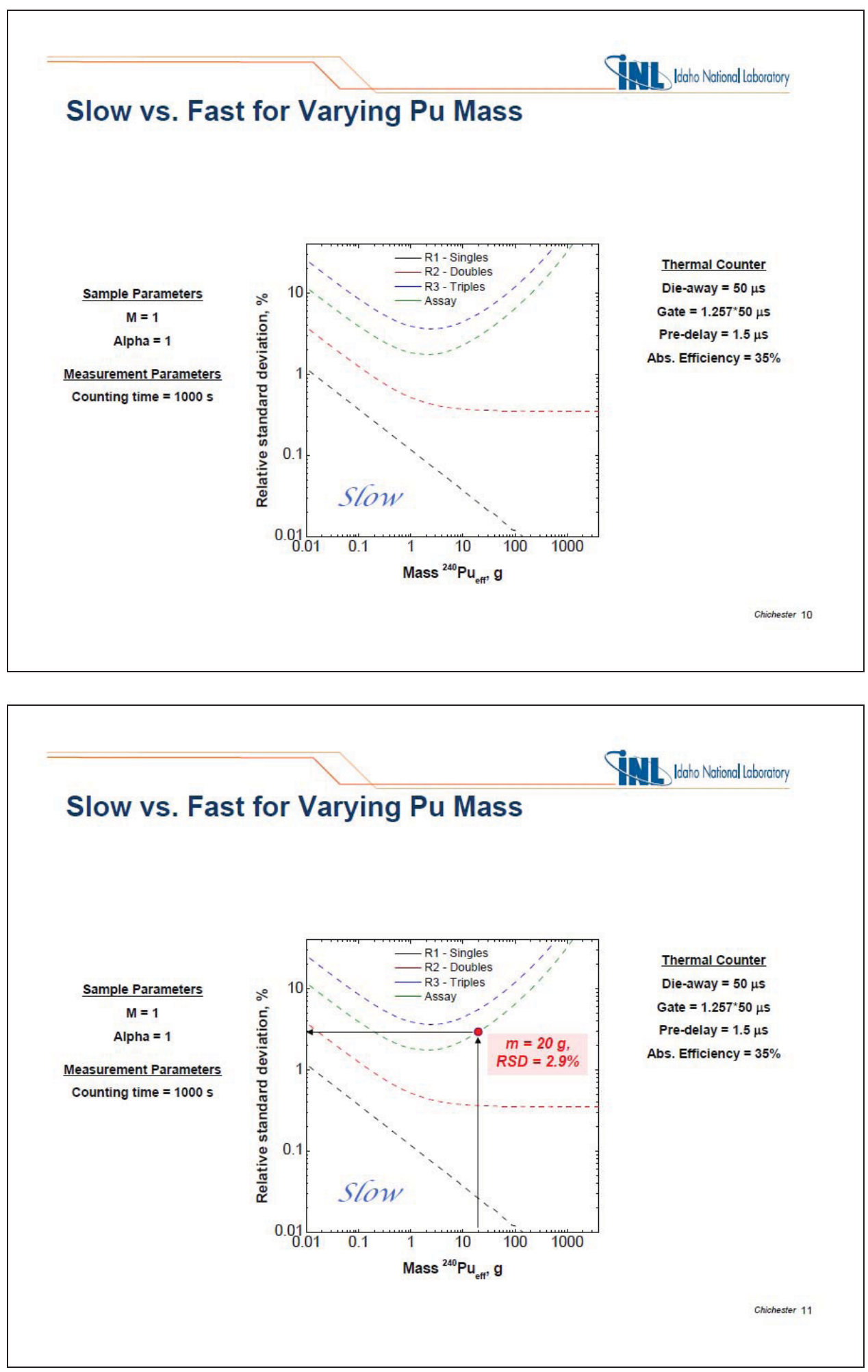

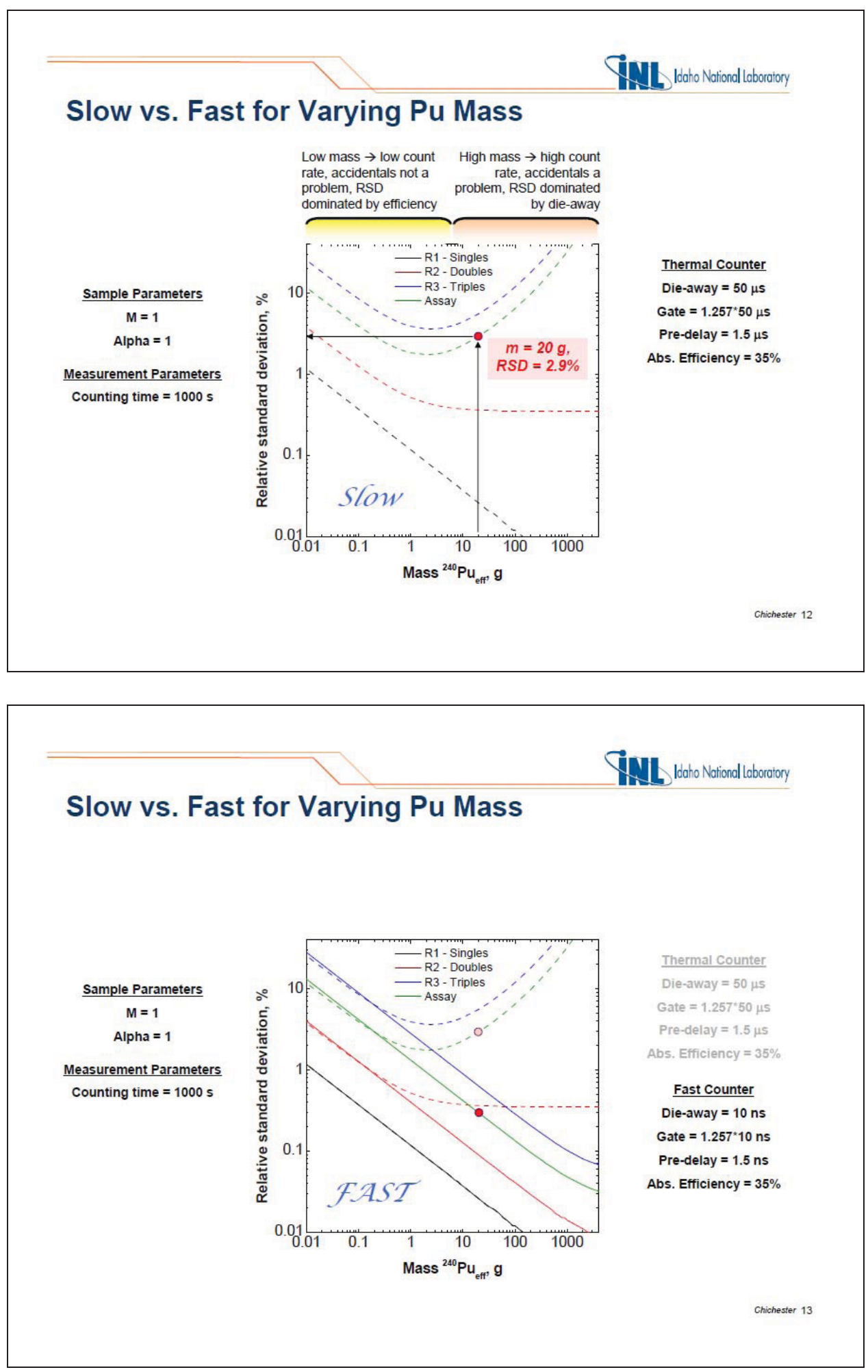


\section{Slow vs. Fast for Varying Pu Mass}

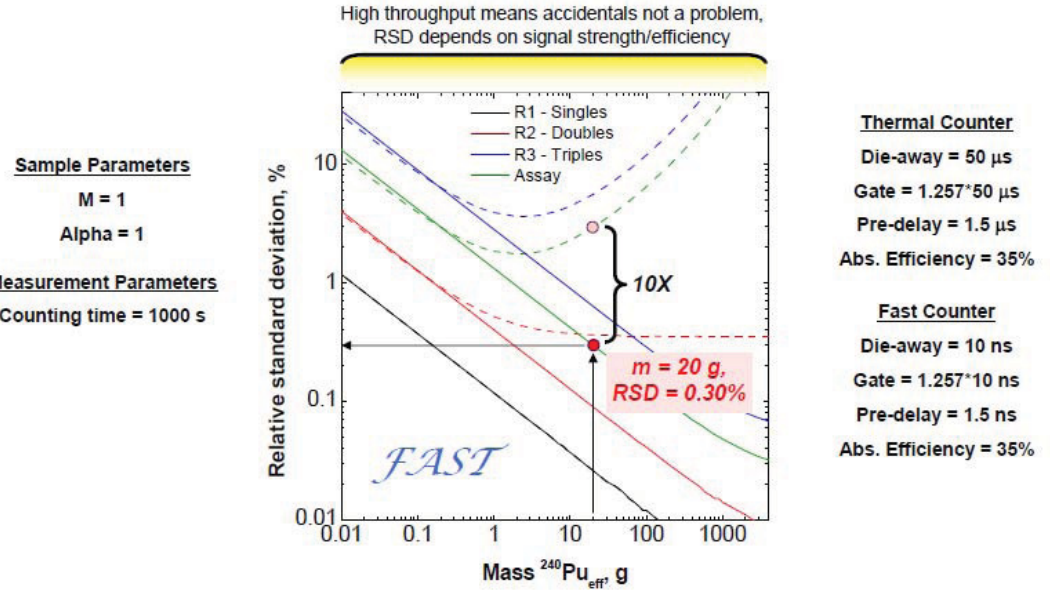

\section{Prior Work - Fast-Neutron Multiplicity (w/ U. Michigan)}

- For four years INL has collaborated with the University of Michigan (S. A. Pozzi, $\mathrm{PI}$ ) to explore the use of liquid scintillator (LS) arrays to assay nuclear material.

- Support towards two graduate students.

Publication of results: 2 journal articles, 6 conference proceedings, 3 INL reports.

- Experiments:

At INL using canisters of MOX fuel.

- At U. Michigan using Cf-252 to characterize LS-detector light output and to develop/integrate data acquisition methods.

- At JRC Ispra using a time-tagged neutron generator and an AmLi source to assay uranium standards, a PWR fresh fuel assembly, and MOX fuel.

- Simulations:

At U. Michigan to benchmark/evaluate the MCNP-PoliMi code and to plan and interpret our experimental work.

At INL to develop approaches for using MCNP5/6 and for parametric studies.
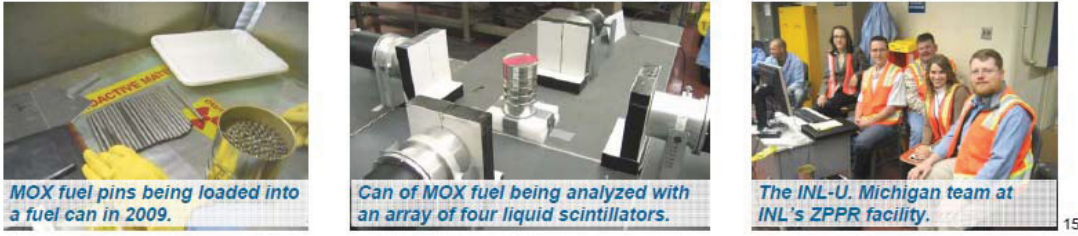

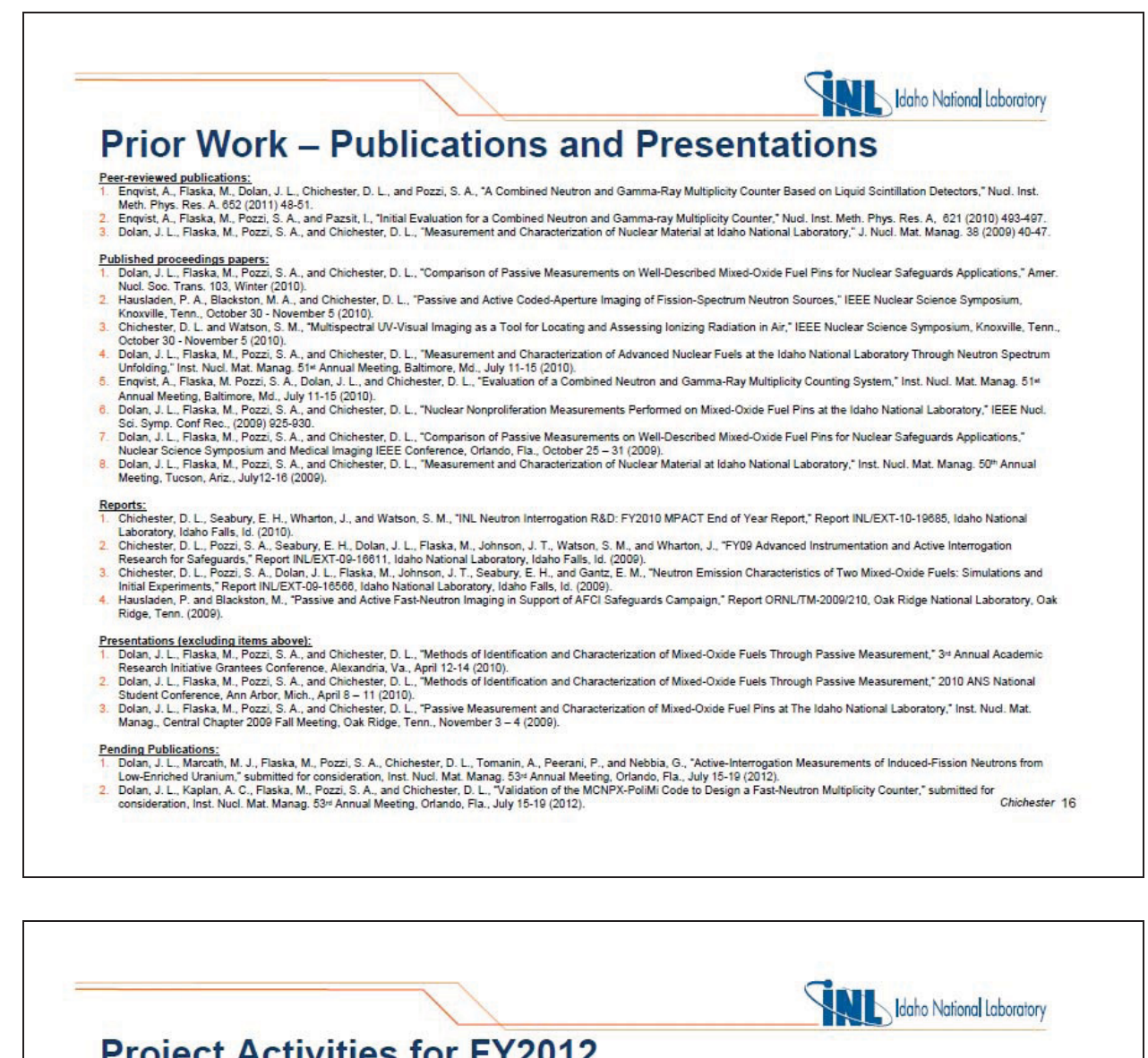

\section{Project Activities for FY2012}

- Simulation and modeling to support development of a prototype fast-neutron multiplicity analysis instrument.

- Explore parameter space using models.

- Define instrument requirements.

- Develop conceptual instrument layout.

- Expand modeling capabilities to include MCNP5/6, to facilitate supercomputer use; compare with newly-released MCNPX-PoliMi.

- Simulate fast neutron multiplicity counter performance expectations and compare with other technologies.

- Experimental activity at U. Michigan and JRC Ispra. - Continue research to develop higher-fidelity light-yield curves for EJ-309 to improve accuracy of models (some work at Ohio U. ).

- Perform lab-scale analyses with ${ }^{252} \mathrm{Cf}$ to improve DAQ implementation using new CAEN digitizers (U. Michigan).

- Perform new set of experiments using kg-range of Pu masses, to validate modeling that correlates doubles rate vs. Pu mass. (U. Michigan team at JRC Ispra).

- Development activity at INL with ORNL support.

- Expand the INL-ORNL collaboration to leverage ORNL expertise related to large detector-array data acquisition systems.

- Begin testing of prototype, multi-board DAQ system with on-board PSD to support year-2 activities at INL.

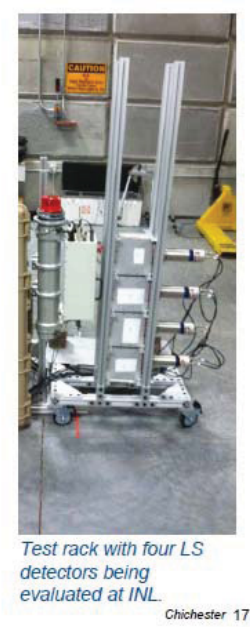



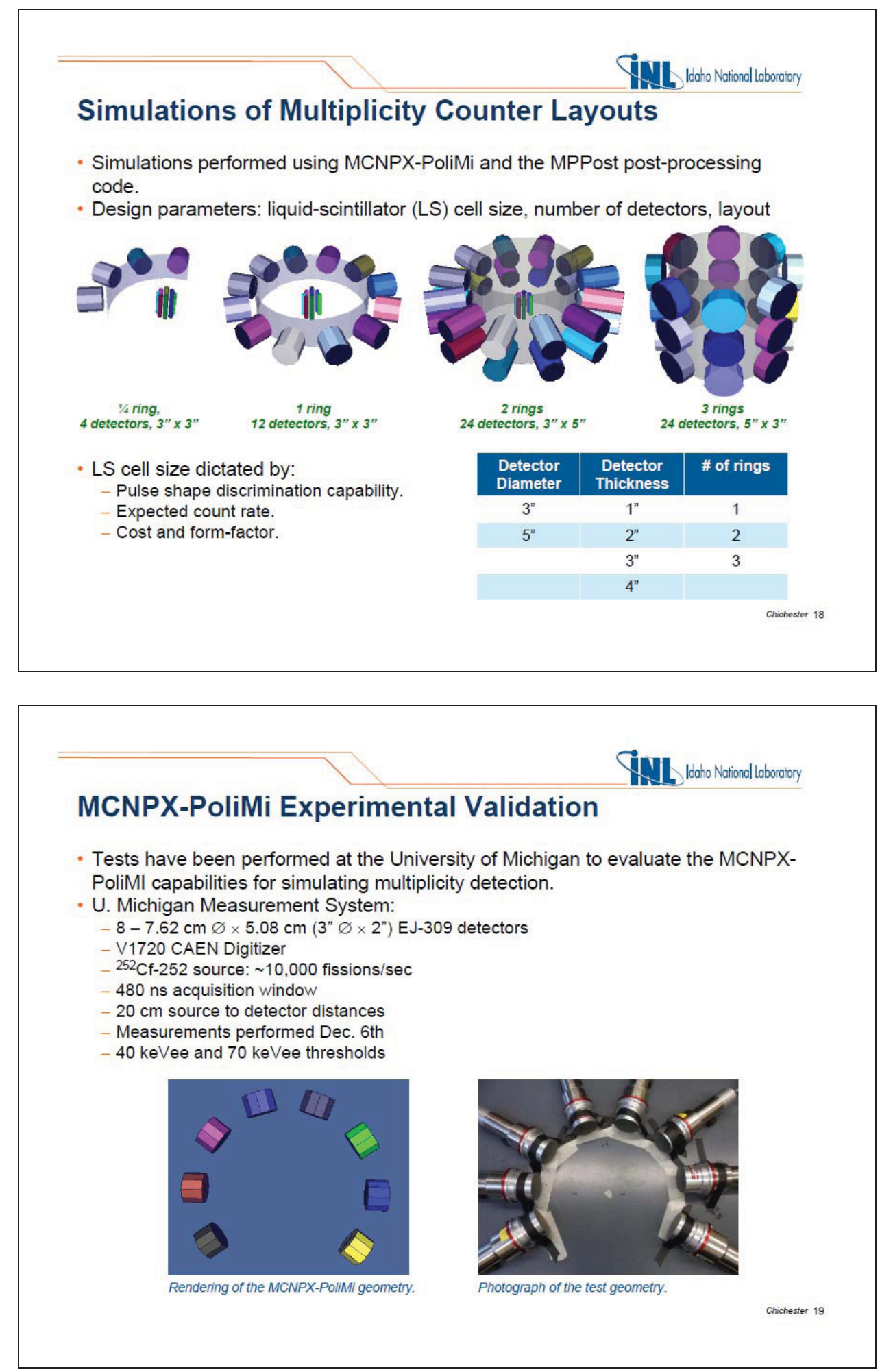
MCNPX-PoliMi Experimental Validation - Thresholds

- Tests were performed to choose the lowest possible pulse height level to use.

- The lower the level, the more counts are kept; $\rightarrow$ more efficiency.

- At lower levels, pulse shape discrimination (PSD) begins to fail.

- With the U. Michigan measurement system 40 keVee is the lowest acceptable pulse level. Two levels were chosen for analysis.
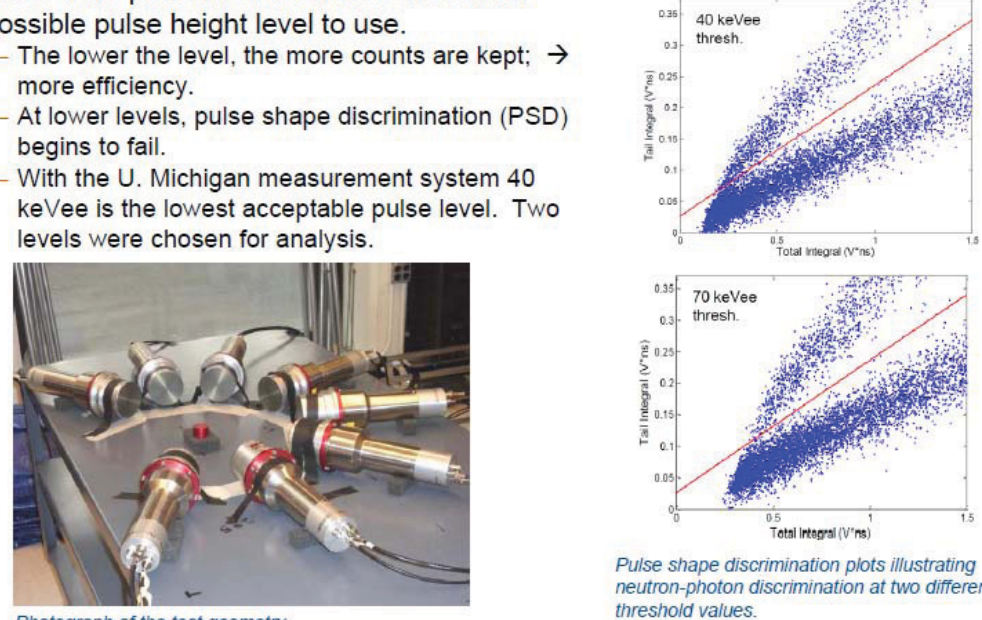

Pulse shape discrimination plots illustrating neutron-photon discrimination at two different

MCNPX-PoliMi Experimental Validation - Comparisons
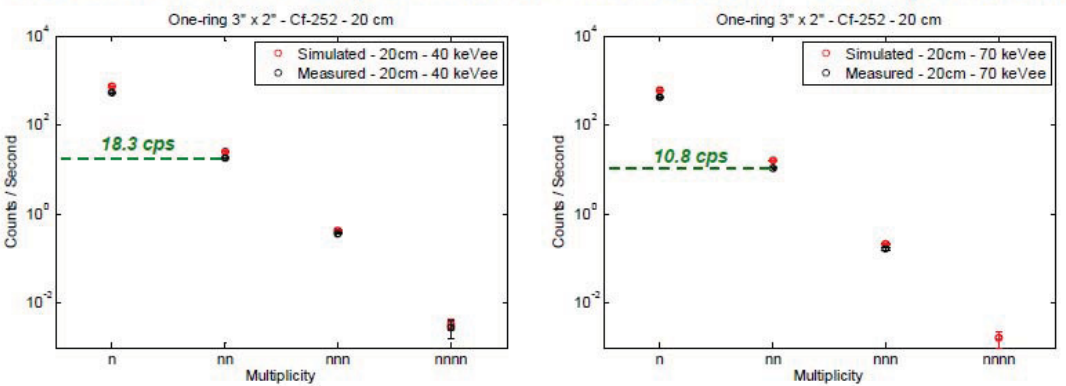

\begin{tabular}{cccc} 
Rates & Measured & Simulated & \% Diff. \\
\hline $\mathrm{n}$ & 547 & 762 & 39.4 \\
$\mathrm{nn}$ & 18.3 & 25.4 & 38.6 \\
$\mathrm{nnn}$ & 0.362 & 0.430 & 19.0 \\
Nnnn & 0.003 & 0.003 & 20
\end{tabular}

\begin{tabular}{cccc} 
Rates & Measured & Simulated & $\%$ Diff. \\
\hline $\mathrm{n}$ & 424 & 616 & 45.3 \\
$\mathrm{nn}$ & 10.8 & 16.0 & 48.1 \\
$\mathrm{nnn}$ & 0.166 & 0.212 & 27.8 \\
$\mathrm{nnnn}$ & 0 & 0.002 & NA
\end{tabular}



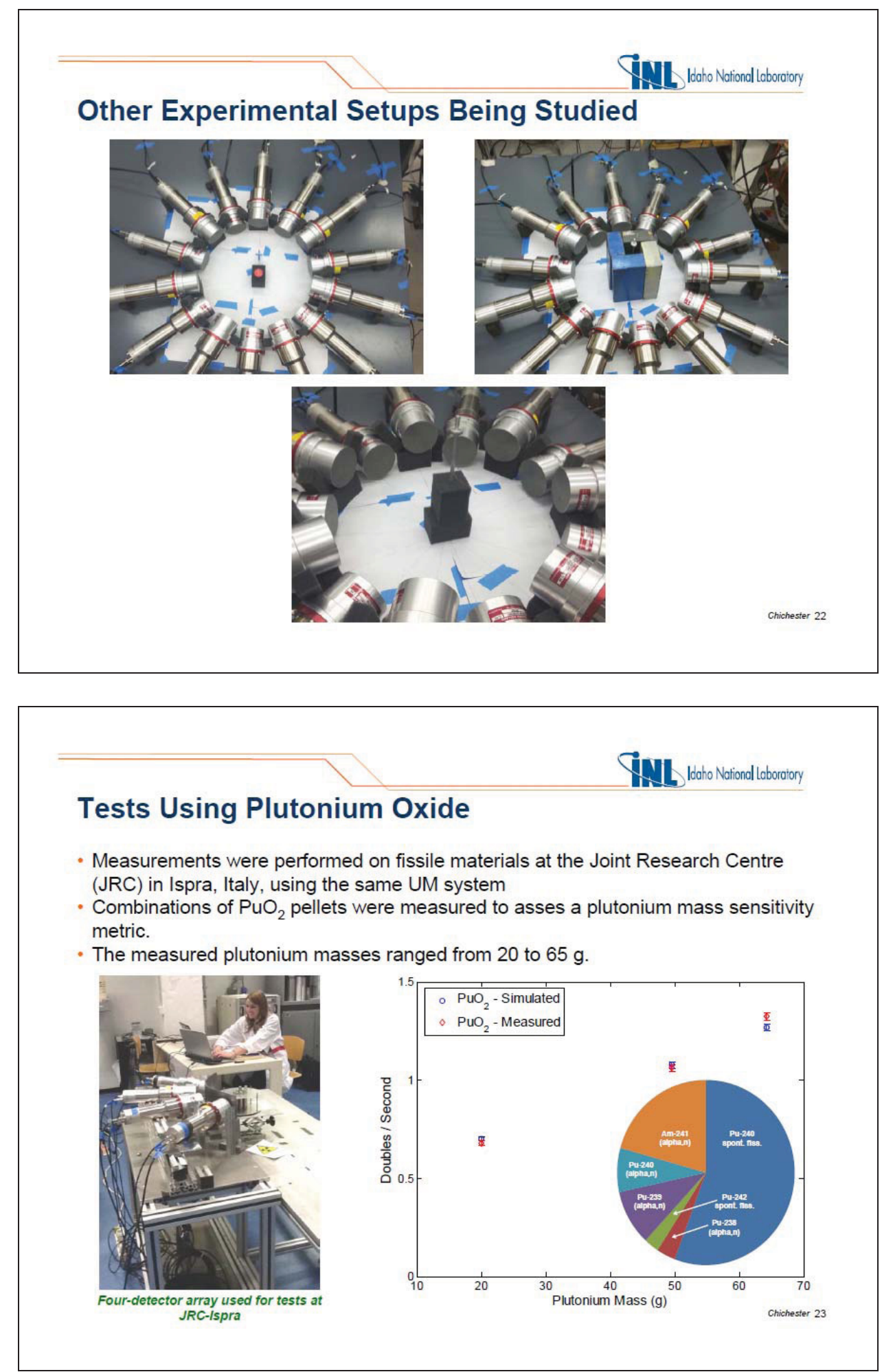

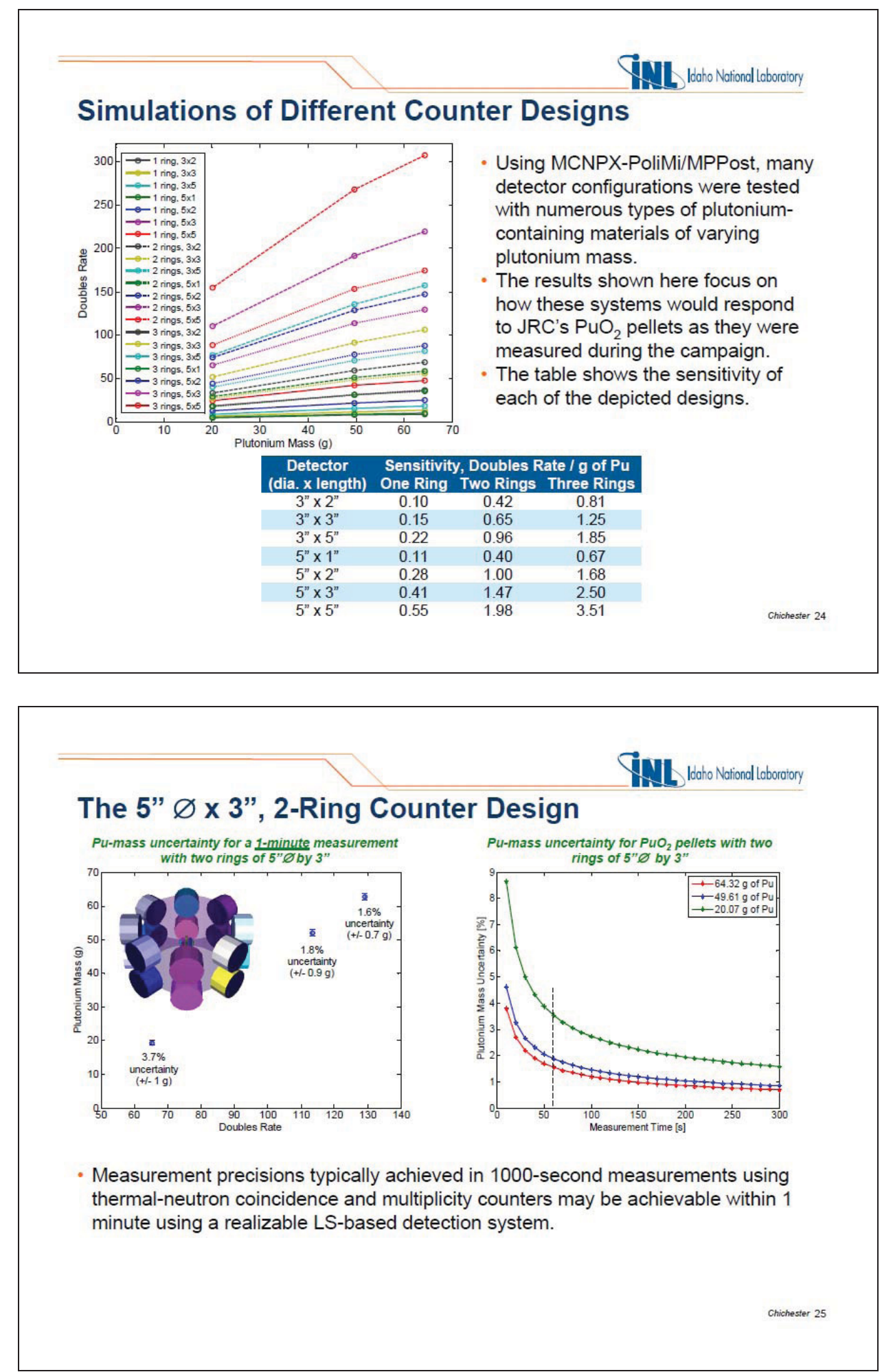


\section{The 5" $\varnothing \times$ 3", 2-Ring Counter Design}

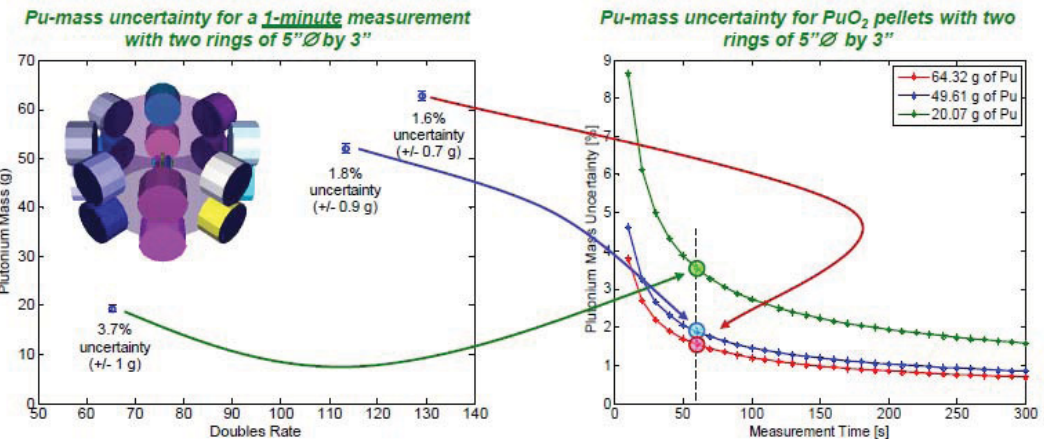

- Measurement precisions typically achieved in 1000-second measurements using thermal-neutron coincidence and multiplicity counters may be achievable within 1 minute using a realizable LS-based detection system.

\section{Future Work - Fast Neutron Multiplicity Counter}

- Complete conceptual design for fast-neutron multiplicity counter.

- Characterizing cross-talk with bench top measurements.

- Testing data-analysis algorithms to minimize cross-talk contribution to measured doubles and triples.

- Optimization of detector placement relative to other detectors.

- Simulations of the potential benefit of additional detectors placed on top of the system. - Estimation of total throughput (neutrons plus photons) per detector node for realistic fuel materials to help choose detector cell size.

- Development work (depending upon budget):

- Assembly and testing of scalable high-speed data acquisition system. - Purchase and test suite of candidate LS detectors for partial array.

- Simulation work:

- Develop high-fidelity models of the conceptual design to be assembled and tested in FY2013 (may need to use existing detectors from other projects).

- Simulate experiments to estimate performance and formulate test plans.

Evaluation of excluding nearest-neighbor doubles as a method to assess/reject cross-talk.

- Evaluation of using neutron-event pulse height as a coincidence gate to reduce cross-talk.

- Evaluation of using fission asymmetry as an additional metric. 


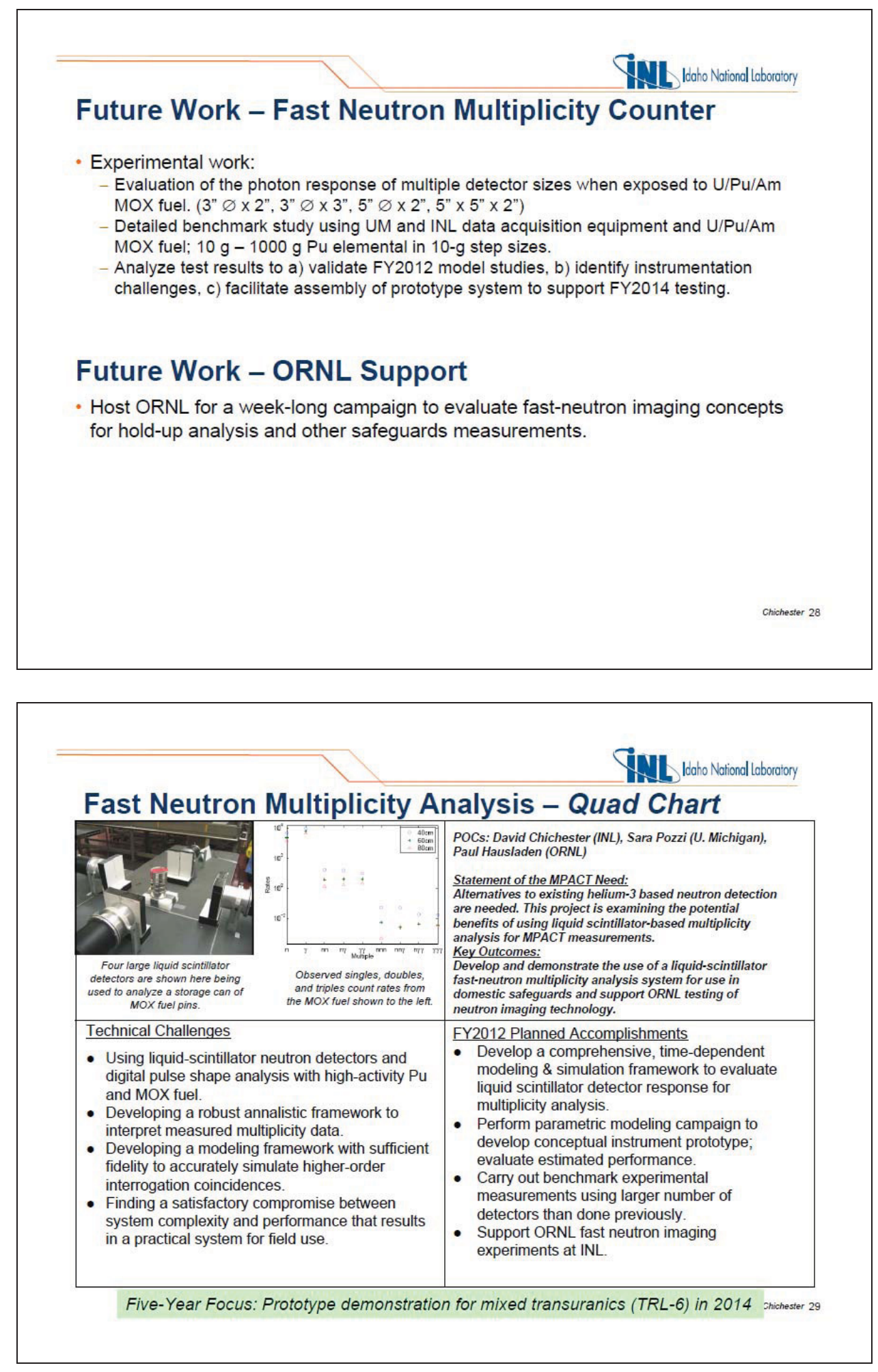




\section{Fast-Neutron Multiplicity Analysis}

D. L. Chichester, M. T. Kinlaw, J. T. Johnson, and S. J.

Thompson (Idaho National Laboratory)

J. L. Dolan, A. C. Kaplan, M. Flaska, A. Enqvist, and S. A.

Pozzi (U. Michigan)

P. A. Hausladen, R. J. Newby, and M. Blackston (ORNL)

For further information contact me at:

Nuclear Nonproliferation Division

Idaho National Laboratory

2525 North Fremont Avenue

Idaho Falls, Idaho 83415

david.chichester@inl.gov

208-526-8920 \& 208-526-9810 